\title{
Quantum estimates in two variable forms for Simpson-type inequalities considering generalized $\Psi$-convex functions with applications
}

https://doi.org/10.1515/phys-2021-0031

received November 20, 2020; accepted April 24, 2021

\section{Introduction}

In order to calculate the derivatives of real functions, classical calculus employs limits. On the other hand, the calculus without limits is known as quantum calculus or $\check{q}$-calculus. According to history, Euler derived the fundamental formulations in $\check{q}$-calculus in the eighteenth century. However, Jackson [1] was the first to develop the conceptions of the definite $\check{q}$-derivative and $\check{q}$-integral. In addition, Andrews [2] examined and inspected a number of studies on quantum calculus. The aforementioned consequences stimulated a more intense discussion of quantum theory in the twentieth century.

The evolution of the research of $\check{q}$-calculus has been presented by its potential utilities in cosmology, multiple hypergeometric functions, Bernoulli and Euler polynomials, Mock theta functions, and more specifically in the study of analytic and harmonic univalent functions. The consensus of scholars who employ $\check{q}$-calculus are physicists, see [3,4]. Baxter [5] presented the exact solutions of numerous frameworks in statistical mechanics. Bettaibi and Mezlini formulated certain $\check{q}$-heat and $\check{q}$-wave equations as in ref. [6]. Moreover, several researchers, including Andrews [2], Gauchman [7], and Kac and Cheung [8] have been compensated for their efforts in proving and proposing new definitions and formulations. The topic of $\check{q}$-theory has become a remarkable trend for many scientists in recent years, and novel results have been explored in previous research [9,10]. Numerous special function theories [11,12] are being assembled within the context of $\check{q}$-calculus, mechanothermodynamics, translimiting states, and generalization of experimental data to analyze the quantum calculus in respect of general energy states $[13,14]$.

With the assistance of special functions and convexity theory, we aim to create the applications of the $\check{q}$-calculus to modify Simpson-type inequality in a twovariable formulation. However, in light of the $\check{q}$-calculus, 
the current article could be the first to examine special functions with the correlation of two-variable formulation.

Convex functions have significant applications in a variety of interesting and engrossing fields of study, and they have also played a pivotal role, including coding theory, optimization, physics, information theory, engineering, and inequality theory. Mathematicians have proposed various novel versions of convex functions in the relevant literature [15-17].

In ref. $[18,19]$, Jensen introduced this property as follows:

Definition 1.1. Let $\mathcal{I} \subseteq \mathbb{R}$ and a mapping $\mathcal{G}: \mathcal{I} \mapsto \mathbb{R}$ is said to be convex on $\mathcal{I}$, if the inequality

$$
\mathcal{G}((1-\zeta) x+\zeta y) \leq(1-\zeta) \mathcal{G}(x)+\zeta \mathcal{G}(y)
$$

holds $\forall x, y \in \mathcal{I}$ and $\zeta \in[0,1]$.

In the field of applied analysis, mathematical inequalities are considered as a prevalent mechanism for collecting descriptive and analytical description. A consistent increase in significance has evolved to address the prerequisites for widespread use of these variants [20-22]. Furthermore, various generalizations have been established by several authors that involve convex functions, such as Hermite-Hadamard, Trapezoid type, Opial, Ostrowski, Grüss, and the supremely illustrious Simpson's inequality.

A mapping $\mathcal{G}:\left[\eta_{1}, \eta_{2}\right] \rightarrow \mathbb{R}$ is four times continuously differentiable and $\left\|\mathcal{G}^{(4)}\right\|_{\infty}=\sup _{z \in\left(\eta_{1}, \eta_{2}\right)}\left|\mathcal{G}^{(4)}(z)\right|<\infty$. Then, one has the following inequality:

$$
\begin{aligned}
& \left|\frac{1}{3}\left[\frac{\mathcal{G}\left(\eta_{1}\right)+\mathcal{G}\left(\eta_{2}\right)}{2}+2 \mathcal{G}\left(\frac{\eta_{1}+\eta_{2}}{2}\right),-\frac{1}{\eta_{2}-\eta_{1}} \int_{\eta_{1}}^{\eta_{2}} \mathcal{G}(z) \mathrm{d} z\right]\right| \\
& \quad \leq \frac{\left(\eta_{2}-\eta_{1}\right)^{4}}{2880}\left\|\mathcal{G}^{(4)}\right\|_{\infty} .
\end{aligned}
$$$$
\frac{\mathcal{G}\left(\eta_{1}, \frac{\eta_{3}+\eta_{4}}{2}\right)+\mathcal{G}\left(\eta_{2}, \frac{\eta_{3}+\eta_{4}}{2}\right)+4 \mathcal{G}\left(\frac{\eta_{1}+\eta_{2}}{2}, \frac{\eta_{3}+\eta_{4}}{2}\right)+\mathcal{G}\left(\frac{\eta_{1}+\eta_{2}}{2}, \eta_{3}\right)+\mathcal{G}\left(\frac{\eta_{1}+\eta_{2}}{2}, \eta_{4}\right)}{9}
$$$$
+\frac{\mathcal{G}\left(\eta_{1}, \eta_{3}\right)+\mathcal{G}\left(\eta_{2}, \eta_{3}\right)+\mathcal{G}\left(\eta_{1}, \eta_{4}\right)+\mathcal{G}\left(\eta_{2}, \eta_{4}\right)}{36}-\frac{1}{6\left(\eta_{2}-\eta_{1}\right)} \int_{\eta_{1}}^{\eta_{2}}\left[\mathcal{G}\left(x, \eta_{3}\right)+4 \mathcal{G}\left(x, \frac{\eta_{3}+\eta_{4}}{2}\right)+\mathcal{G}\left(x, \eta_{4}\right)\right]{ }_{0} d_{\check{q}_{1}} x
$$$$
-\frac{1}{6\left(\eta_{4}-\eta_{3}\right)} \int_{\eta_{3}}^{\eta_{4}}\left[\mathcal{G}\left(\eta_{1}, y\right)+4 \mathcal{G}\left(\frac{\eta_{1}+\eta_{2}}{2}, y\right)+\mathcal{G}\left(\eta_{2}, y\right)\right]{ }_{0} d_{\check{q}_{2}} y+\frac{1}{\left(\eta_{2}-\eta_{1}\right)\left(\eta_{4}-\eta_{3}\right)} \int_{\eta_{1} \eta_{3}}^{\eta_{2} \eta_{4}} \mathcal{G}(x, y)_{0} d_{\check{q}_{2}} y_{0} d_{\check{q}_{1}} x
$$$$
=\left(\eta_{2}-\eta_{1}\right)\left(\eta_{4}-\eta_{3}\right) \int_{0}^{1} \int_{0}^{1} \Omega_{1}\left(\zeta_{1}, \check{q}_{1}\right) \Omega_{2}\left(\zeta_{2}, \check{q}_{2}\right) \frac{\eta_{1}, \eta_{3} \partial_{\check{q}_{1}, \check{q}_{2}}^{2} \mathcal{G}\left(\left(1-\zeta_{1}\right) \eta_{1}+\zeta_{1} \eta_{2},\left(1-\zeta_{2}\right) \eta_{3}+\zeta_{2} \eta_{4}\right)}{\eta_{1} \partial_{\check{q}_{1}} \zeta_{1 \eta_{3}} \partial_{\check{q}_{2}} \zeta_{2}} d_{\check{q}_{1}} \zeta_{10} d_{\check{q}_{2}} \zeta_{2},
$$

For further generalizations, modifications, and developments, we refer to refs [23-25] and references cited therein. One of the used frames of reference corresponds to the "strongly convex functions." The origin of that term is the generalization of convex functions, contemplated by Polyak [26]. Its incentives are accessible in optimization theory and many other related fields. In ref. [27], Karamardian used this functional class to discover the existence of a solution for nonlinear complementarity problems. $\mathrm{Zu}$ and Marcotte [28] have applied the aforesaid class to obtain the convergence analysis of the iterative methods for solving variational inequalities and equilibrium problems. Nikodem and Pales [29] proposed a correlation of inner product spaces with strongly convex functions as a new and novel concept with concrete utilities as a follow-up. The primal dual gradient dynamical approach with exponential stability has been investigated by Qu and Li [30]. Rashid et al. [31] proposed Hermite-Hadamard-type inequalities for various classes of strongly convex functions, which provide upper and lower bounds for the integrand. For further presentations on real-world phenomena, we refer to refs. [32-34].

Our intention is to establish the novel $\check{q}$-integral identity of Simpson-type within a class of generalized $\Psi$-convex functions in two variable forms. Kalsoom et al. [35] established the quantum integral Simpson type inequality for convex function on co-ordinates as follows:

Lemma 1.1. [35] Assume that a mapping $\mathcal{G}: \Delta \subseteq \mathbb{R}^{2} \rightarrow \mathbb{R}$ having a mixed partial $\check{q}_{1} \check{q}_{2}$-differentiable function defined on $\Delta^{\mathrm{o}}$ (the interior of $\Delta$ ) with $\frac{\eta_{1}, \eta_{3} \partial_{\bar{q}_{1}, \breve{q}_{2}}^{2} h(z, w)}{\eta_{1} \partial_{\breve{q}_{1}} z_{\eta_{3}} \mathrm{\partial}_{q_{2}} w}$ to be continuous and integrable on $\left[\eta_{1}, \eta_{2}\right] \times\left[\eta_{3}, \eta_{4}\right] \subset \Delta^{\circ}$ with $0<q_{i}<1$ and $1 \leq i \leq 2$, then 
where

$$
\Omega_{1}\left(\zeta_{1}, \check{q}_{1}\right)= \begin{cases}\check{q}_{1} \zeta_{1}-\frac{1}{6}, & \zeta_{1} \in\left[0, \frac{1}{2}\right), \\ \check{q}_{1} \zeta_{1}-\frac{5}{6}, & \zeta_{1} \in\left[\frac{1}{2}, 1\right),\end{cases}
$$

and

$$
\Omega_{2}\left(\zeta_{2}, \check{q}_{2}\right)= \begin{cases}\check{q}_{2} \zeta_{2}-\frac{1}{6}, & \zeta_{2} \in\left[0, \frac{1}{2}\right), \\ \check{q}_{2} \zeta_{2}-\frac{5}{6}, & \zeta_{2} \in\left[\frac{1}{2}, 1\right) .\end{cases}
$$

This article investigates and presents a novel idea of higher-order strongly generalized $\Psi$-convex and quasiconvex functions in the sense of Raina's function. Considering the novel auxiliary identity that correlates with the Raina function and the $\check{q}$-calculus theory, numerous new Simpson-type inequalities are apprehended via the aforesaid classes of functions derived in two variable forms. Additionally, this suggested scheme in $\check{q}$-calculus theory connected with Definitions 2.5 and 2.7 introduced new results for Simpson-type inequalities in hypergeometric and Mittag-Leffler sense. Finally, our findings may stimulate further investigation into special relativity theory and quantum theory.

\section{Prelude}

Let $\mathcal{K}$ be a non-empty closed set in $\mathbb{R}^{n}$ and $\mathcal{G}: \mathcal{K} \mapsto \mathbb{R}$ a continuous function.

Noor [36] introduced a class of non-convex mappings known as $\Psi$-convex functions.

Definition 2.1. [36] A mapping $\mathcal{G}: \mathcal{K} \mapsto \mathbb{R}$ on the $\Psi$ convex set $\mathcal{K}$ is said to be $\Psi$-convex, if

$$
\begin{aligned}
& \mathcal{G}\left(x+\zeta e^{i \Psi}(y-x)\right) \leq(1-\zeta) \mathcal{G}(x)+\zeta \mathcal{G}(y), \\
& \zeta \in[0,1], \forall x, y \in \mathcal{K} .
\end{aligned}
$$

Observe that every convex mapping is $\Psi$-convex, but converse does not hold in general.

In ref. [37], Raina contemplated the subsequent class of function

$$
\mathcal{F}_{\sigma, \lambda}^{\vartheta}(t)=\mathcal{F}_{\sigma, \lambda}^{\vartheta(0), \vartheta(1), \ldots}(t)=\sum_{p=0}^{\infty} \frac{\vartheta(p)}{\Gamma(\sigma p+\lambda)} t^{p},
$$

where $\gamma, \rho>0,|t|<\mathbb{R}$, and

$$
\vartheta=(\vartheta(0), \vartheta(1), \ldots \vartheta(p), \ldots)
$$

is a bounded sequence of $\mathbb{R}^{+}$. Moreover, take $\sigma=1, \lambda=0$ in (2.2) and

$$
\vartheta(p)=\frac{\left(\beta_{1}\right)_{p}\left(\beta_{2}\right)_{p}}{\left(\beta_{3}\right)_{p}} \quad \text { for } p=0,1,2,3 \ldots,
$$

where the parameters $\beta_{i}(i=1,2,3)$ as if it were real or complex (assuming $\beta_{3}=0,-1,-2, \ldots$ ), and the symbol $(z)_{p}$ specified by

$$
\begin{aligned}
(z)_{p} & =\frac{\Gamma(z+p)}{\Gamma(z)}=z(z+1) \ldots(z+p-1), \\
p & =0,1,2, \ldots,
\end{aligned}
$$

and its domain is restricted as $|t| \leq 1$ (with $t \in \mathbb{C}$ ), then we get the hypergeometric function as follows:

$$
\mathcal{F}_{\sigma, \lambda}^{\vartheta}(t)=F\left(\beta_{1} ; \beta_{2} ; \beta_{3} ; t\right)=\sum_{p=0}^{\infty} \frac{\left(\beta_{1}\right)_{p}\left(\beta_{2}\right)_{p}}{p !\left(\beta_{3}\right)_{p}} t^{p} .
$$

furthermore, if $\vartheta=(1,1, \ldots)$ with $\sigma=\beta_{1},\left(\Re\left(\beta_{1}\right)>0\right), \lambda=1$ and its domain is restricted as $t \in \mathbb{C}$ in equation (2.2), then we get the Mittag-Leffler function as follows:

$$
\bar{E}_{\beta_{1}}(t)=\sum_{p=0}^{\infty} \frac{1}{\Gamma\left(1+\beta_{1} p\right)} t^{p} .
$$

Next, we evoke a new class of set and a new class of functions, including Raina's functions.

Definition 2.2. A non-empty set $\mathcal{K}_{\mathcal{F}}$ is said to be generalized $\Psi$-convex set, if

$$
x+\mathcal{F}_{\sigma, \lambda}^{\vartheta}(y-x) \in \mathcal{K}_{\mathcal{F}}
$$

for all $x, y \in \mathcal{K}_{\mathcal{F}}, \zeta \in[0,1]$.

We now define the generalized $\Psi$-convex function presented by Vivas-Cortez et al. [38].

Definition 2.3. [38] Let a set $\mathcal{K}_{\overline{\mathcal{F}}} \subseteq \mathbb{R}$ and we say that a function $\mathcal{G}: \mathcal{K}_{\mathcal{F}} \rightarrow \mathbb{R}$ is generalized $\Psi$-convex, if

$$
\mathcal{G}\left(x+\zeta \mathcal{F}_{\sigma, \lambda}^{\vartheta}(y-x)\right) \leq(1-\zeta) \mathcal{G}(x)+\zeta \mathcal{G}(y)
$$

for all $x, y \in \mathcal{K}_{\mathcal{F}}, \zeta \in[0,1]$.

Definition 2.4. We say that a function $\mathcal{G}: \mathcal{K}_{\mathcal{F}} \rightarrow \mathbb{R}$ is higher order strongly generalized $\Psi$-convex having $\delta \geq 0$, if

$$
\begin{aligned}
& \mathcal{G}\left(x+\zeta \mathcal{F}_{\sigma, \lambda}^{\vartheta}(y-x)\right) \\
& \quad \leq(1-\zeta) \mathcal{G}(x)+\zeta \mathcal{G}(y)-\delta \zeta(1-\zeta)\left\|\mathcal{F}_{\sigma, \lambda}^{\vartheta}(y-x)\right\|^{\theta}
\end{aligned}
$$

for all $x, y \in \mathcal{K}_{\mathcal{F}}, \zeta \in[0,1]$ and $\theta>0$. 
Some remarkable special cases are discussed as follows:

I. Taking $\delta=0$, then Definition 2.5 reduces to Definition 2.3.

II. Taking $\theta=0$, then the generalized higher-order strongly $\Psi$-convex mappings reduces to generalized strongly $\Psi$-convex mappings, that is,

Definition 2.5. We say that a function $G: \mathcal{K}_{\mathcal{F}} \rightarrow \mathbb{R}$ is higher order strongly generalized $\Psi$-convex having $\delta \geq 0$, if

$$
\begin{aligned}
& \mathcal{G}\left(x+\zeta \mathcal{F}_{\sigma, \lambda}^{\vartheta}(y-x)\right) \\
& \quad \leq(1-\zeta) \mathcal{G}(x)+\zeta \mathcal{G}(y)-\delta \zeta(1-\zeta)\left\|\mathcal{F}_{\sigma, \lambda}^{\vartheta}(y-x)\right\|^{2}
\end{aligned}
$$

for all $x, y \in \mathcal{K}_{\mathcal{F}}, \zeta \in[0,1]$

III. Letting $\mathcal{F}_{\sigma, \lambda}^{\vartheta}(y-x)=y-x$, then Definition 2.5 changes to higher-order strongly convex mappings.

Definition 2.6. We say that a function $\mathcal{G}: \mathcal{K} \rightarrow \mathbb{R}$ is higher order strongly convex having $\delta \geq 0$, if

$$
\begin{aligned}
& \mathcal{G}((1-\zeta) x+\zeta y) \\
& \quad \leq(1-\zeta) \mathcal{G}(x)+\zeta \mathcal{G}(y)-\delta \zeta(1-\zeta)\|(y-x)\|^{2}
\end{aligned}
$$

for all $x, y \in \mathcal{K}, \zeta \in[0,1]$.

Definition 2.7. We say that a function $\mathcal{G}: \mathcal{K}_{\mathcal{F}} \rightarrow \mathbb{R}$ is higher order strongly generalized $\Psi$-quasi-convex having $\delta \geq 0$, if

$$
\begin{aligned}
& \mathcal{G}\left(x+\zeta \mathcal{F}_{\sigma, \lambda}^{\vartheta}(y-x)\right) \\
& \quad \leq \max \{\mathcal{G}(x), \mathcal{G}(y)\}-\delta \zeta(1-\zeta)\left\|\mathcal{F}_{\sigma, \lambda}^{\vartheta}(y-x)\right\|^{\theta}
\end{aligned}
$$

for all $x, y \in \mathcal{K}_{\mathcal{F}}, \zeta \in[0,1]$ and $\theta>0$.

For an exceptional appropriate selections of the Raina's function $\mathcal{F}_{\sigma, \lambda}^{\vartheta}(.,),. \delta$ and $\theta$, one can attain several earlier and new classes of higher-order generalized strongly convex and quasi-convex mappings. This demonstrates that the new idea involving Raina's function is wide and modifying one.

In addition, we highlight some key concepts and definitions in the $\check{q}$-analog for one and two-variables.

Let $\mathcal{J}=\left[\zeta_{1}, \zeta_{2}\right] \subseteq \mathbb{R}$, and let $\mathcal{U}=\left[\zeta_{1}, \zeta_{2}\right] \times\left[\zeta_{3}, \zeta_{4}\right] \subseteq \mathbb{R}^{2}$ with constants $\check{q}, \check{q}_{i} \in(0,1), i=1,2$.

In ref. $[39,40]$, authors investigated the notions of $\check{q}$-derivative, $\check{q}$-integral, and their features for finite interval, which has been demonstrated as
Definition 2.8. Suppose that $\mathcal{G}: \mathcal{J} \rightarrow \mathbb{R}, t \in \mathcal{J}$ is a continuous mapping, then one has $\check{q}$-derivative of $\mathcal{G}$ on $\mathcal{J}$ at $t$ which is written as

$$
\zeta_{1} \mathcal{D}_{\tilde{q}} \mathcal{G}(t)=\frac{\mathcal{G}(t)-\mathcal{G}\left(q t+(1-q) \zeta_{1}\right)}{(1-q)\left(t-\zeta_{1}\right)}, \quad t \neq \zeta_{1} .
$$

It can be observed that

$$
\lim _{t \rightarrow \zeta_{1}} \mathcal{D}_{\check{q}} \mathcal{G}(t)=\zeta_{1} \mathcal{D}_{\check{q}} \mathcal{G}\left(\zeta_{1}\right)
$$

which implies that the mapping $\mathcal{G}$ is $\check{q}$-differentiable over $\mathcal{J}$, also $\zeta_{1} \mathcal{D}_{\check{q}} \mathcal{G}(t)$ exists $\forall t \in \mathcal{J}$.

It is noted that if $\zeta_{1}=0$ in equation (2.11), then ${ }_{0} \mathcal{D}_{\check{q}} \mathcal{G}=\mathcal{D}_{\check{q}} \mathcal{G}$, where $\mathcal{D}_{\check{q}} \mathcal{G}$ is well-defined $\check{q}$-derivative of $\mathcal{G}(t)$, i.e., is mentioned as

$$
\mathcal{D}_{\check{q}} \mathcal{G}(t)=\frac{\mathcal{G}(t)-\mathcal{G}(q t)}{(1-q)(t)} .
$$

Definition 2.9. Suppose that $\mathcal{G}: \mathcal{J} \rightarrow \mathbb{R}$ is a continuous function, indicated as $\zeta_{1} \mathcal{D}_{\tilde{q}}^{2} \mathcal{G}$, provided that $\zeta_{1} \mathcal{D}_{\tilde{q}}^{2} \mathcal{G}$ be $\check{q}$-differentiable from $\mathcal{J} \rightarrow \mathbb{R}$ identified by

$$
\zeta_{1} \mathcal{D}_{\check{q}}^{2} \mathcal{G}=\zeta_{1} \mathcal{D}_{\check{q}}\left(\zeta_{1} \mathcal{D}_{\check{q}} \mathcal{G}\right)
$$

Thus, $\zeta_{1} \mathcal{D}_{\check{q}}^{j} \mathcal{G}: \mathcal{J} \rightarrow \mathbb{R}$ denotes the higher order $\check{q}$-differentiable function.

Definition 2.10. Suppose that $\mathcal{G}: \mathcal{J} \rightarrow \mathbb{R}$ is a continuous function and the $\check{q}$-integral on $\mathcal{J}$ is expressed as

$$
\begin{aligned}
& \int_{\zeta_{1}}^{t} \mathcal{G}(z)_{\zeta_{1}} d_{\check{q}^{\prime}} z=(1-\check{q})\left(t-\zeta_{1}\right) \sum_{j=0}^{\infty} \check{q}^{j} \mathcal{G}\left(\check{q}^{j} t+\left(1-\check{q}^{j}\right) \zeta_{1}\right), \\
& \quad \forall t \in \mathcal{J} .
\end{aligned}
$$

Next, if $\zeta_{1}=0$ in equation (2.12), so there is an integral formulation of $\check{q}$, which is signified as

$$
\int_{0}^{t} \mathcal{G}(z)_{0} d_{\check{q}} z=(1-\check{q}) t \sum_{j=0}^{\infty} \check{q}^{j} \mathcal{G}\left(\check{q}^{j} t\right) \text {. }
$$

Theorem 2.1. Suppose that $\mathcal{G}: \mathcal{J} \rightarrow \mathbb{R}$ is a continuous function, then the subsequent assertions fulfill:

(i) $\zeta_{1} \mathcal{D}_{\check{q}} \int_{\zeta_{1}}^{t} \mathcal{G}(z)_{\zeta_{1}} d_{\check{q}} z=\mathcal{G}(t)$;

(ii) $\int_{\zeta_{1}}^{t} \zeta_{1} \mathcal{D}_{\breve{q}} \mathcal{G}(z)_{\zeta_{1}} d_{\breve{q}} z=\mathcal{G}(t)$;

(iii) $\int_{\zeta_{2}}^{t} \zeta_{1} \mathcal{D}_{\check{q}} \mathcal{G}(z)_{\zeta_{1}} d_{\breve{q}} z=\mathcal{G}(t)-\mathcal{G}\left(\zeta_{2}\right), \quad \zeta_{2} \in\left(\zeta_{1}, t\right)$. 
Theorem 2.2. Suppose that $\mathcal{G}: \mathcal{J} \rightarrow \mathbb{R}$ is a continuous function and $a \in \mathbb{R}$, then the subsequent assertions fulfill:

(i) $\int_{\zeta_{1}}^{t}\left[\mathcal{G}_{1}(z)+\mathcal{G}_{2}(z)\right]_{\zeta_{1}} d_{\ddot{q}} z=\int_{\zeta_{1}}^{t} \mathcal{G}_{1}(z)_{\zeta_{1}} d_{\ddot{q}} z+\int_{\zeta_{1}}^{t} \mathcal{G}_{2}(z)_{\zeta_{1}} d_{\ddot{q}} z$;

(ii) $\int_{\zeta_{1}}^{t}\left(a \mathcal{G}_{1}(z)\right)_{\zeta_{1}} d_{\ddot{q}} z=a \int_{\zeta_{1}}^{t} \mathcal{G}_{1}(z)_{\zeta_{1}} d_{\ddot{q}} z$.

In ref. [35], Kalsoom et al. presented the quantum integral identities in a two-variable context as follows:

Definition 2.11. [35] Suppose a mapping in two-variables sense $\mathcal{G}: \mathcal{U} \rightarrow \mathbb{R}$ is continuous, then the partial $\check{q}_{1} \check{q}_{2}$ and $\check{q}_{1} \check{q}_{2}$-derivative at $(z, w) \in\left[\zeta_{1}, \zeta_{2}\right] \times\left[\zeta_{3}, \zeta_{4}\right]$ are, respectively, described as:

$$
\begin{aligned}
\frac{\zeta_{1} \partial_{\check{q}_{1}} \mathcal{G}(z, w)}{\zeta_{1} \partial_{\check{q}_{1}} z}= & \frac{\mathcal{G}(z, w)-\mathcal{G}\left(\check{q}_{1} z+\left(1-\check{q}_{1}\right) \zeta_{1}, w\right)}{\left(1-\check{q}_{1}\right)\left(z-\zeta_{1}\right)}, \\
\frac{\zeta_{3} \partial_{\check{q}_{2}} \mathcal{G}(z, w)}{\zeta_{3} \partial_{\check{q}_{2}} w}= & \frac{\mathcal{G}(z, w)-\mathcal{G}\left(z, \check{q}_{2} w+\left(1-\check{q}_{2}\right) \zeta_{3}\right)}{\left(1-\check{q}_{2}\right)\left(w-\zeta_{3}\right)}, \\
w \neq \zeta_{3}, & \\
\frac{\zeta_{1} \zeta_{3} \partial_{\check{q}_{1}, \check{q}_{2}}^{2} \mathcal{G}(z, w)}{\zeta_{1} \partial_{\check{q}_{1}} z_{\zeta_{3}} \partial_{\check{q}_{2}} w}= & \frac{1}{\left(1-\check{q}_{1}\right)\left(1-\check{q}_{2}\right)\left(z-\zeta_{1}\right)\left(w-\zeta_{3}\right)} \\
& \times\left[\mathcal{G}\left(\check{q}_{1} z+\left(1-\check{q}_{1}\right) \zeta_{1}, \check{q}_{2} w+\left(1-\check{q}_{2}\right) \zeta_{3}\right)\right. \\
& -\mathcal{G}\left(\check{q}_{1} z+\left(1-\check{q}_{1}\right) \zeta_{1}, w\right) \\
& \left.-\mathcal{G}\left(z, \check{q}_{2} w+\left(1-\check{q}_{2}\right) \zeta_{3}\right)+\mathcal{G}(z, w)\right], \\
& z \neq \zeta_{1}, w \neq \zeta_{3} .
\end{aligned}
$$

We say that a function $\mathcal{G}: \mathcal{U} \rightarrow \mathbb{R}$ is partially $\check{q}_{1}, \check{q}_{2}$ and $\check{q}_{1} \check{q}_{2}$-differentiable on $\left[\zeta_{1}, \zeta_{2}\right] \times\left[\zeta_{3}, \zeta_{4}\right]$ if $\frac{\zeta_{\zeta_{1}} \check{q}_{1} \mathcal{G}(z, w)}{\zeta_{1} \check{q}_{1} z}, \frac{\zeta_{3} \check{q}_{2} \mathcal{G}(z, w)}{\zeta_{3} \check{\check{q}}_{2} w}$

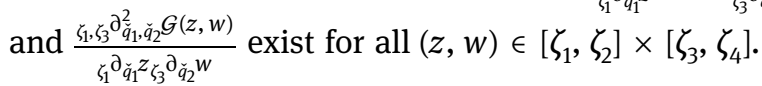

Definition 2.12. Suppose a function in two-variables sense $\mathcal{G}: \mathcal{U} \rightarrow \mathbb{R}$ is continuous, then the definite $\check{q}_{1} \check{q}_{2}$ integral on $\left[\zeta_{1}, \zeta_{2}\right] \times\left[\zeta_{3}, \zeta_{4}\right]$ is stated as

$$
\begin{aligned}
& \int_{\zeta_{3}}^{t} \int_{\zeta_{1}}^{t} \mathcal{G}(z, w)_{\zeta_{1}} d_{\check{q}_{1}} z_{\zeta_{3}} d_{\check{q}_{2}} w \\
& =\left(1-\check{q}_{1}\right)\left(1-\check{q}_{2}\right)\left(t-\zeta_{1}\right)\left(t_{1}-\zeta_{3}\right) \sum_{k=0}^{\infty} \sum_{j=0}^{\infty} \check{q}_{1}^{j} \check{q}_{2}^{K} \mathcal{G}\left(\check{q}_{1}^{j} t\right. \\
& \left.\quad+\left(1-\check{q}_{1}^{j}\right) \zeta_{1}, \check{q}_{2}^{K} t_{1}+\left(1-\check{q}_{2}^{K}\right) \zeta_{3}\right)
\end{aligned}
$$

for $\left(t, t_{1}\right) \in\left[\zeta_{1}, \zeta_{2}\right] \times\left[\zeta_{3}, \zeta_{4}\right]$.
Theorem 2.3. Suppose a function in two-variables sense $\mathcal{G}: \mathcal{B} \rightarrow \mathbb{R}$ is continuous, then the subsequent assertions fulfill:

(i) $\frac{\zeta_{1} \zeta_{3}}{\zeta_{1} \partial_{\check{q}_{1}} t_{\zeta_{1}} \partial_{\zeta_{3}} \partial_{\check{q}_{2}} t_{1}} \int_{\zeta_{4}}^{t_{1}} \int_{\zeta_{1}}^{t} \mathcal{G}(z, w)_{\zeta_{1}} d_{\check{q}_{1}} z_{\zeta_{3}} d_{\check{q}_{2}} w=\mathcal{G}\left(t, t_{1}\right)$;

(ii) $\int_{\zeta_{3}}^{t_{1}} \int_{\zeta_{1}}^{t} \frac{\zeta_{1}, \zeta_{3} \partial_{\check{q}_{1}}^{2} \check{q}_{2} \mathcal{G}(z, w)}{\zeta_{1} \partial_{\check{q}_{1}} z_{\zeta_{3}} \partial_{\check{q}_{2}} w} \zeta_{1} d_{\check{q}_{1}} z_{\zeta_{3}} d_{\check{q}_{2}} w=\mathcal{G}\left(t, t_{1}\right)$;

(iii) $\int_{t_{2}}^{t_{1}} \int_{y_{1}}^{t} \frac{\zeta_{1} \zeta_{\zeta} \partial_{\check{q}_{1}, \check{q}_{2}}^{2} \mathcal{G}(z, w)}{\zeta_{1} \partial_{\check{q}_{1}} z_{\zeta_{3}} \partial_{\check{q}_{2}} w} \zeta_{1} d_{\check{q}_{1}} z_{\zeta_{3}} d_{\check{q}_{2}} w ;=\mathcal{G}\left(t, t_{1}\right)$

$-\mathcal{G}\left(t, t_{2}\right)-\mathcal{G}\left(y_{1}, t_{1}\right)+\mathcal{G}\left(y_{1}, t_{2}\right), \quad\left(y_{1}, t_{2}\right) \in\left(\zeta_{1}, t\right) \times\left(\zeta_{4}, t_{1}\right)$.

Theorem 2.4. Suppose that $\mathcal{G}_{1}, \mathcal{G}_{2}: \mathcal{U} \rightarrow \mathbb{R}$ are continuous mappings of two-variables. Then the subsequent assertions fulfill for $\left(t, t_{1}\right) \in\left[\zeta_{1}, \zeta_{2}\right] \times\left[\zeta_{3}, \zeta_{4}\right]$,

$$
\begin{aligned}
& \text { (i) } \int_{\zeta_{3}}^{t_{1}} \int_{\zeta_{1}}^{t}\left[\mathcal{G}_{1}(z, w)+\mathcal{G}_{2}(z, w)\right]_{\zeta_{1}} d_{\check{q}_{1}} z_{\zeta_{4}} d_{\check{q}_{2}} w \\
& =\int_{\zeta_{3}}^{t_{1}} \int_{\zeta_{1}}^{t} \mathcal{G}_{1}(z, w)_{\zeta_{1}} d_{\check{q}_{1}} z_{\zeta_{3}} d_{\check{q}_{2}} w+\int_{\zeta_{3}}^{t} \int_{\zeta_{1}}^{t} \mathcal{G}_{2}(z, w)_{\zeta_{1}} d_{\check{q}_{1}} z_{\zeta_{3}} d_{\check{q}_{2}} w ; \\
& \text { (ii) } \int_{\zeta_{\zeta}}^{t_{1}} \int_{\zeta_{1}}^{t} a \mathcal{G}(z, w)_{\zeta_{1}} d_{\check{q}_{1}} z_{\zeta_{3}} d_{\check{q}_{2}} w=a \int_{\zeta_{\zeta}}^{t_{\zeta_{1}}} \int_{\zeta_{1}}^{t} \mathcal{G}(z, w)_{\zeta_{1}} d_{\check{q}_{1}} z_{\zeta_{3}} d_{\check{q}_{2}} w \text {. }
\end{aligned}
$$

\section{A $\check{q}_{1} \check{q}_{2}$-integral identity for generalized $\Psi$-convex functions associated with Raina's function}

To illustrate the important consequences of this article, we proceed with some integral identities and inequalities for generalized $\Psi$-convex functions with the well-known Raina function.

Throughout this investigation, we utilized the following hypothesis:

(I) Let $\sigma, \lambda>0$ and $\vartheta=(\vartheta(0), \ldots, \vartheta(p))$ is a bounded sequence of positive real numbers.

(II) Suppose that a twice partial $\check{q}_{1} \check{q}_{2}$-differentiable map$\operatorname{ping} \mathcal{G}: \widetilde{O} \subseteq \mathbb{R}^{2} \rightarrow \mathbb{R}$ defined on $\widetilde{O}^{\circ}$ (the interior of $\widetilde{O}$ )

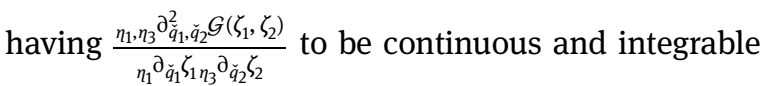
on $\left[\eta_{1}, \eta_{1}+\mathcal{F}_{\sigma, \lambda}^{g}\left(\eta_{2}-\eta_{1}\right)\right] \times\left[\eta_{3}, \eta_{3}+\mathcal{F}_{\sigma, \lambda}^{9}\left(\eta_{4}-\eta_{3}\right)\right] \subset \widetilde{O}^{\circ}$ such that $\mathcal{F}_{\sigma, \lambda}^{\vartheta}\left(\eta_{2}-\eta_{1}\right), \mathcal{F}_{\sigma, \lambda}^{9}\left(\eta_{4}-\eta_{3}\right)>0$ for $0<\check{q}_{1}$, $\check{q}_{2}<1$. 
Lemma 3.1. Suppose that Assumptions (I) and (II) are satisfied, then the following equality holds:

$$
\begin{aligned}
& \mathcal{G}\left(\eta_{1}, \frac{2 \eta_{3}+\mathcal{F}_{\sigma, \lambda}^{9}\left(\eta_{4}-\eta_{3}\right)}{2}\right)+\mathcal{G}\left(\eta_{1}+\mathcal{F}_{\sigma, \lambda}^{9}\left(\eta_{2}-\eta_{1}\right), \frac{2 \eta_{3}+\mathcal{F}_{\sigma, \lambda}^{9}\left(\eta_{4}-\eta_{3}\right)}{2}\right) \\
& \left.\widetilde{\Theta}_{\mathcal{G}}\left(\eta_{1}, \eta_{2}, \eta_{3}, \eta_{4} ; \check{q}_{1}, \check{q}_{2}\right)=\frac{1}{9}\right)+4 \mathcal{G}\left(\frac{2 \eta_{1}+\mathcal{F}_{\sigma, \lambda}^{9}\left(\eta_{2}-\eta_{1}\right)}{2}, \frac{2 \eta_{3}+\mathcal{F}_{\sigma, \lambda}^{9}\left(\eta_{4}, \eta_{3}\right)}{2}\right)+\mathcal{G}\left(\frac{2 \eta_{1}+\mathcal{F}_{\sigma, \lambda}^{9}\left(\eta_{2}-\eta_{1}\right)}{2}, \eta_{3}\right) \\
& +\mathcal{G}\left(\frac{2 \eta_{1}+\mathcal{F}_{\sigma, \lambda}^{9}\left(\eta_{2}-\eta_{1}\right)}{2}, \eta_{3}+\mathcal{F}_{\sigma, \lambda}^{9}\left(\eta_{4}-\eta_{3}\right)\right) \\
& +\frac{1}{36}\left[\begin{array}{c}
\mathcal{G}\left(\eta_{1}, \eta_{3}\right)+\mathcal{G}\left(\eta_{1}+\mathcal{F}_{\sigma, \lambda}^{9}\left(\eta_{2}-\eta_{1}\right), \eta_{3}\right)+\mathcal{G}\left(\eta_{1}, \eta_{3}+\mathcal{F}_{\sigma, \lambda}^{9}\left(\eta_{4}-\eta_{3}\right)\right) \\
+\mathcal{G}\left(\eta_{1}+\mathcal{F}_{\sigma, \lambda}^{9}\left(\eta_{2}-\eta_{1}\right), \eta_{3}+\mathcal{F}_{\sigma, \lambda}^{g}\left(\eta_{4}-\eta_{3}\right)\right)
\end{array}\right] \\
& -\frac{1}{6 \mathcal{F}_{\sigma, \lambda}^{g}\left(\eta_{2}-\eta_{1}\right)} \int_{\eta_{1}}^{\eta_{1}+\mathcal{F}_{\sigma, \lambda}^{g}\left(\eta_{2}-\eta_{1}\right)}\left[\mathcal{G}\left(x, \eta_{3}\right)+4 \mathcal{G}\left(x, \frac{2 \eta_{3}+\mathcal{F}_{\sigma, \lambda}^{g}\left(\eta_{4}-\eta_{3}\right)}{2}\right)\right. \\
& \left.+\mathcal{G}\left(x, \eta_{3}+\mathcal{F}_{\sigma, \lambda}^{9}\left(\eta_{4}-\eta_{3}\right)\right)\right]{ }_{0} d_{\check{q}_{1}} x \\
& -\frac{1}{6 \mathcal{F}_{\sigma, \lambda}^{9}\left(\eta_{4}, \eta_{3}\right)} \int_{\eta_{3}}^{\eta_{3}+\mathcal{F}_{\sigma, \lambda}^{9}\left(\eta_{4}, \eta_{3}\right)}\left[\mathcal{G}\left(\eta_{1}, y\right)+4 \mathcal{G}\left(\frac{2 \eta_{1}+\mathcal{F}_{\sigma, \lambda}^{9}\left(\eta_{2}-\eta_{1}\right)}{2}, y\right)\right. \\
& \left.+\mathcal{G}\left(\eta_{1}+\mathcal{F}_{\sigma, \lambda}^{\vartheta}\left(\eta_{2}-\eta_{1}\right), y\right)\right]{ }_{0} d_{\check{q}_{2}} y \\
& +\frac{1}{\mathcal{F}_{\sigma, \lambda}^{g}\left(\eta_{2}-\eta_{1}\right) \mathcal{F}_{\sigma, \lambda}^{g}\left(\eta_{4}-\eta_{3}\right)} \int_{\eta_{1}}^{\eta_{1}+\mathcal{F}_{\sigma, \lambda}^{g}\left(\eta_{2}-\eta_{1}\right)} \int_{\eta_{3}}^{\eta_{3}+\mathcal{F}_{\sigma, \lambda}^{g}\left(\eta_{4}-\eta_{3}\right)} \mathcal{G}(x, y)_{0} d_{\check{q}_{2}} y_{0} d_{\check{q}_{1}} x \\
& =\mathcal{F}_{\sigma, \lambda}^{9}\left(\eta_{2}-\eta_{1}\right) \mathcal{F}_{\sigma, \lambda}^{9}\left(\eta_{4}-\eta_{3}\right) \int_{0}^{1} \int_{0}^{1} \Omega_{1}\left(\zeta_{1}, \check{q}_{1}\right) \Omega_{2}\left(\zeta_{2}, \check{q}_{2}\right) \\
& \times \frac{\eta_{1}, \eta_{3} \partial_{\check{q}_{1}, \check{q}_{2}}^{2} \mathcal{G}\left(\eta_{1}+\zeta_{1} \mathcal{F}_{\sigma, \lambda}^{g}\left(\eta_{2}-\eta_{1}\right), \eta_{3}+\zeta_{2} \mathcal{F}_{\sigma, \lambda}^{g}\left(\eta_{4}-\eta_{3}\right)\right)}{\eta_{1} \partial_{\check{q}_{1}} \zeta_{1 \eta_{3}} \partial_{\check{q}_{2}} \zeta_{2}} d_{\check{q}_{1}} \zeta_{10} d_{\check{q}_{2}} \zeta_{2},
\end{aligned}
$$

where

$$
\begin{gathered}
\Omega_{1}\left(\zeta_{1}, \check{q}_{1}\right)= \begin{cases}\check{q}_{1} \zeta_{1}-\frac{1}{6}, & \text { if } 0 \leq \zeta_{1}<\frac{1}{2}, \\
\check{q}_{1} \zeta_{1}-\frac{5}{6}, & \text { if } \frac{1}{2} \leq \zeta_{1} \leq 1,\end{cases} \\
\Omega_{2}\left(\zeta_{2}, \check{q}_{2}\right)= \begin{cases}\check{q}_{2} \zeta_{2}-\frac{1}{6}, & \text { if } 0 \leq \zeta_{2}<\frac{1}{2}, \\
\check{q}_{2} \zeta_{2}-\frac{5}{6}, & \text { if } \frac{1}{2} \leq \zeta_{2} \leq 1 .\end{cases}
\end{gathered}
$$


DE GRUYTER

Quantum estimates in two variable forms for Simpson-type inequalities

311

Proof. In view of definition of partial $\check{q}_{1} \check{q}_{2}$-derivatives and definite $\check{q}_{1} \check{q}_{2}$-integrals, one has

$$
\begin{aligned}
& \int_{0}^{\frac{1}{2}} \int_{0}^{\frac{1}{2}}\left(\check{q}_{1} \zeta_{1}-\frac{1}{6}\right)\left(\check{q}_{2} \zeta_{2}-\frac{1}{6}\right) \frac{\eta_{1}, \eta_{3} \partial_{\check{q}_{1}, \breve{q}_{2}}^{2} \mathcal{G}\left(\eta_{1}+\zeta_{1} \mathcal{F}_{\sigma, \lambda}^{\Theta}\left(\eta_{2}-\eta_{1}\right), \eta_{3}+\zeta_{2} \mathcal{F}_{\sigma, \lambda}^{\Theta}\left(\eta_{4}-\eta_{3}\right)\right)}{\eta_{1} \partial_{\check{q}_{1}} \zeta_{1} \eta_{3} \partial_{\check{q}_{2}} \zeta_{2}}{ }_{0} d_{\check{q}_{1}} \zeta_{10} d_{\check{q}_{2}} \zeta_{2} \\
& +\int_{0}^{\frac{1}{2}} \int_{\frac{1}{2}}^{1}\left(\check{q}_{1} \zeta_{1}-\frac{1}{6}\right)\left(\check{q}_{2} \zeta_{2}-\frac{5}{6}\right) \frac{\eta_{1}, \eta_{3} \partial_{\check{q}_{1} \check{q}_{2}}^{2} \mathcal{G}\left(\eta_{1}+\zeta_{1} \mathcal{F}_{\sigma, \lambda}^{9}\left(\eta_{2}-\eta_{1}\right), \eta_{3}+\zeta_{2} \mathcal{F}_{\sigma, \lambda}^{g}\left(\eta_{4}-\eta_{3}\right)\right)}{\eta_{1} \partial_{\check{q}_{1}} \zeta_{1 \eta_{3}} \partial_{\check{q}_{2}} \zeta_{2}}{ }_{0} d_{\check{q}_{1}} \zeta_{10} d_{\check{q}_{2}} \zeta_{2} \\
& +\int_{\frac{1}{2}}^{1} \int_{0}^{1}\left(\check{q}_{1} \zeta_{1}-\frac{5}{6}\right)\left(\check{q}_{2} \zeta_{2}-\frac{1}{6}\right) \frac{\eta_{1}, \eta_{3} \partial_{\check{q}_{1}, \breve{q}_{2}}^{2} \mathcal{G}\left(\eta_{1}+\zeta_{1} \mathcal{F}_{\sigma, \lambda}^{\vartheta}\left(\eta_{2}-\eta_{1}\right), \eta_{3}+\zeta_{2} \mathcal{F} \sigma_{\sigma, \lambda}\left(\eta_{4}-\eta_{3}\right)\right)}{\eta_{1} \partial_{\check{q}_{1}} \zeta_{1} \partial_{\eta_{3}} \partial_{\check{q}_{2}} \zeta_{2}}{ }_{0} d_{\check{q}_{1}} \zeta_{10} d_{\check{q}_{2}} \zeta_{2}
\end{aligned}
$$

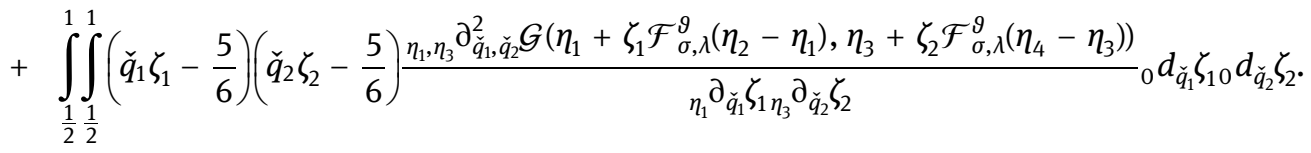

By the definition of partial $\check{q}_{1} \check{q}_{2}$-derivatives and deftnite $\check{q}_{1} \check{q}_{2}$-integrals, we have

$$
\begin{aligned}
& \int_{0}^{\frac{1}{2}} \int_{0}^{\frac{1}{2}}\left(\check{q}_{1} \zeta_{1}-\frac{1}{6}\right)\left(\check{q}_{2} \zeta_{2}-\frac{1}{6}\right) \frac{\eta_{1}, \eta_{3} \partial_{\check{q}_{1}, \check{q}_{2}}^{2} \mathcal{G}\left(\eta_{1}+\zeta_{1} \mathcal{F}_{\sigma, \lambda}^{g}\left(\eta_{2}-\eta_{1}\right), \eta_{3}+\zeta_{2} \mathcal{F}_{\sigma, \lambda}^{g}\left(\eta_{4}-\eta_{3}\right)\right)}{\eta_{1} \partial_{\check{q}_{1}} \zeta_{1} \eta_{3} \partial_{\check{q}_{2}} \zeta_{2}} d_{\check{q}_{1}} \zeta_{10} d_{\check{q}_{2}} \zeta_{2} \\
& =\frac{1}{\left(1-\check{q}_{1}\right)\left(1-\check{q}_{2}\right) \mathcal{F}_{\sigma, \lambda}^{9}\left(\eta_{2}-\eta_{1}\right) \mathcal{F}_{\sigma, \lambda}^{9}\left(\eta_{4}-\eta_{3}\right)} \int_{0}^{\frac{1}{2}} \int_{0}^{\frac{1}{2}} \frac{\left(\check{q}_{1} \zeta_{1}-\frac{1}{6}\right)\left(\check{q}_{2} \zeta_{2}-\frac{1}{6}\right)}{\zeta_{1} \zeta_{2}} \\
& \times\left(\mathcal{G}\left(\eta_{1}+\zeta_{1} \check{q}_{1} \mathcal{F}_{\sigma, \lambda}^{9}\left(\eta_{2}-\eta_{1}\right), \eta_{3}+\zeta_{2} \check{q}_{2} \mathcal{F}_{\sigma, \lambda}^{9}\left(\eta_{4}-\eta_{3}\right)\right)-\mathcal{G}\left(\eta_{1}+\zeta_{1} \check{q}_{1} \mathcal{F}_{\sigma, \lambda}^{9}\left(\eta_{2}-\eta_{1}\right), \zeta_{2}\right)\right. \\
& \left.-\mathcal{G}\left(\zeta_{1}, \eta_{3}+\zeta_{2} \check{q}_{2} \mathcal{F}_{\sigma, \lambda}^{9}\left(\eta_{4}-\eta_{3}\right)\right)+\mathcal{G}\left(\zeta_{1}, \zeta_{2}\right)\right)_{0} d_{\check{q}_{1}} \zeta_{10} d_{\check{q}_{2}} \zeta_{2} \text {. }
\end{aligned}
$$

We observe that

$$
\begin{aligned}
& \frac{1}{\mathcal{F}_{\sigma, \lambda}^{g}\left(\eta_{2}-\eta_{1}\right) \mathcal{F}_{\sigma, \lambda}^{g}\left(\eta_{4}-\eta_{3}\right)} \sum_{k=1}^{\infty} \sum_{j=1}^{\infty} \check{q}_{1}^{\kappa} \check{q}_{2}^{j} \mathcal{G}\left(\eta_{1}+\frac{\check{q}_{1}^{K}}{2} \mathcal{F}_{\sigma, \lambda}^{g}\left(\eta_{2}-\eta_{1}\right), \eta_{3}+\frac{\check{q}_{2}^{j}}{2} \mathcal{F}_{\sigma, \lambda}^{g}\left(\eta_{4}-\eta_{3}\right)\right) \\
& =-\frac{1}{\mathcal{F}_{\sigma, \lambda}^{9}\left(\eta_{2}-\eta_{1}\right) \mathcal{F}_{\sigma, \lambda}^{9}\left(\eta_{4}-\eta_{3}\right)} \mathcal{G}\left(\frac{2 \eta_{1}+\mathcal{F}_{\sigma, \lambda}^{9}\left(\eta_{2}-\eta_{1}\right)}{2}, \frac{2 \eta_{3}+\mathcal{F}_{\sigma, \lambda}^{9}\left(\eta_{4}-\eta_{3}\right)}{2}\right) \\
& -\frac{1}{\mathcal{F}_{\sigma, \lambda}^{g}\left(\eta_{2}-\eta_{1}\right) \mathcal{F}_{\sigma, \lambda}^{g}\left(\eta_{4}-\eta_{3}\right)} \sum_{k=0}^{\infty} q_{1}^{\kappa} \mathcal{G}\left(\eta_{1}+\frac{\check{q}_{1}^{\kappa}}{2} \mathcal{F}_{\sigma, \lambda}^{g}\left(\eta_{2}-\eta_{1}\right), \frac{2 \eta_{3}+\mathcal{F}_{\sigma, \lambda}^{9}\left(\eta_{4}-\eta_{3}\right)}{2}\right) \\
& -\frac{1}{\mathcal{F}_{\sigma, \lambda}^{g}\left(\eta_{2}-\eta_{1}\right) \mathcal{F}_{\sigma, \lambda}^{g}\left(\eta_{4}-\eta_{3}\right)} \sum_{j=0}^{\infty} \check{q}_{2}^{j} \mathcal{G}\left(\frac{2 \eta_{1}+\mathcal{F}_{\sigma, \lambda}^{9}\left(\eta_{2}-\eta_{1}\right)}{2}, \eta_{3}+\frac{\check{q}_{2}^{j}}{2} \mathcal{F}_{\sigma, \lambda}^{g}\left(\eta_{4}-\eta_{3}\right)\right) \\
& +\frac{1}{\mathcal{F}_{\sigma, \lambda}^{g}\left(\eta_{2}-\eta_{1}\right) \mathcal{F}_{\sigma, \lambda}^{g}\left(\eta_{4}-\eta_{3}\right)} \sum_{k=0}^{\infty} \sum_{j=0}^{\infty} \check{q}_{1}^{K} \check{q}_{2}^{j} \mathcal{G}\left(\eta_{1}+\frac{\check{q}_{1}^{K}}{2} \mathcal{F}_{\sigma, \lambda}^{g}\left(\eta_{2}-\eta_{1}\right), \eta_{3}+\frac{\check{q}_{2}^{j}}{2} \mathcal{F}_{\sigma, \lambda}^{g}\left(\eta_{4}-\eta_{3}\right)\right), \\
& -\frac{\check{q}_{2}}{\mathcal{F}_{\sigma, \lambda}^{g}\left(\eta_{2}-\eta_{1}\right) \mathcal{F}_{\sigma, \lambda}^{g}\left(\eta_{4}-\eta_{3}\right)} \sum_{k=1}^{\infty} \sum_{j=0}^{\infty} \check{q}_{1}^{\kappa} \check{q}_{2}^{j} \mathcal{G}\left(\eta_{1}+\frac{\check{q}_{1}^{K}}{2} \mathcal{F}_{\sigma, \lambda}^{g}\left(\eta_{2}-\eta_{1}\right), \eta_{3}+\frac{\check{q}_{2}^{j}}{2} \mathcal{F}_{\sigma, \lambda}^{g}\left(\eta_{4}-\eta_{3}\right)\right) \\
& =\frac{\check{q}_{2}}{\mathcal{F}_{\sigma, \lambda}^{g}\left(\eta_{2}-\eta_{1}\right) \mathcal{F}_{\sigma, \lambda}^{g}\left(\eta_{4}-\eta_{3}\right)} \sum_{j=0}^{\infty} \check{q}_{2}^{j} \mathcal{G}\left(\frac{2 \eta_{1}+\mathcal{F}_{\sigma, \lambda}^{g}\left(\eta_{2}-\eta_{1}\right)}{2}, \eta_{3}+\frac{\check{q}_{2}^{j}}{2} \mathcal{F}_{\sigma, \lambda}^{g}\left(\eta_{4}-\eta_{3}\right)\right) \\
& -\frac{\check{q}_{2}}{\mathcal{F}_{\sigma, \lambda}^{g}\left(\eta_{2}-\eta_{1}\right) \mathcal{F}_{\sigma, \lambda}^{g}\left(\eta_{4}-\eta_{3}\right)} \sum_{k=0}^{\infty} \sum_{j=0}^{\infty} \check{q}_{1}^{K} \check{q}_{2}^{j} \mathcal{G}\left(\eta_{1}+\frac{\check{q}_{1}^{K}}{2} \mathcal{F}_{\sigma, \lambda}^{g}\left(\eta_{2}-\eta_{1}\right), \eta_{3}+\frac{\check{q}_{2}^{j}}{2} \mathcal{F}_{\sigma, \lambda}^{g}\left(\eta_{4}-\eta_{3}\right)\right), \\
& -\frac{\check{q}_{1}}{\mathcal{F}_{\sigma, \lambda}^{g}\left(\eta_{2}-\eta_{1}\right) \mathcal{F}_{\sigma, \lambda}^{g}\left(\eta_{4}-\eta_{3}\right)} \sum_{k=0}^{\infty} \sum_{j=1}^{\infty} \check{q}_{1}^{\kappa} \check{q}_{2}^{j} \mathcal{G}\left(\eta_{1}+\frac{\check{q}_{1}^{\kappa}}{2} \mathcal{F}_{\sigma, \lambda}^{g}\left(\eta_{2}-\eta_{1}\right), \eta_{3}+\frac{\check{q}_{2}^{j}}{2} \mathcal{F}_{\sigma, \lambda}^{g}\left(\eta_{4}-\eta_{3}\right)\right)
\end{aligned}
$$


312

— Yu-Ming Chu et al.

DE GRUYTER

$$
\begin{aligned}
& =\frac{\check{q}_{1}}{\mathcal{F}_{\sigma, \lambda}^{g}\left(\eta_{2}-\eta_{1}\right) \mathcal{F}_{\sigma, \lambda}^{g}\left(\eta_{4}-\eta_{3}\right)} \sum_{k=0}^{\infty} \check{q}_{1}^{k} \mathcal{G}\left(\eta_{1}+\frac{\check{q}_{1}^{K}}{2} \mathcal{F}_{\sigma, \lambda}^{g}\left(\eta_{2}-\eta_{1}\right), \frac{2 \eta_{3}+\mathcal{F}_{\sigma, \lambda}^{g}\left(\eta_{4}-\eta_{3}\right)}{2}\right) \\
& -\frac{\check{q}_{1}}{\mathcal{F}_{\sigma, \lambda}^{g}\left(\eta_{2}-\eta_{1}\right) \mathcal{F}_{\sigma, \lambda}^{g}\left(\eta_{4}-\eta_{3}\right)} \sum_{\kappa=0}^{\infty} \sum_{j=0}^{\infty} \check{q}_{1}^{\kappa} \check{q}_{2}^{j} \mathcal{G}\left(\eta_{1}+\frac{\check{q}_{1}^{\kappa}}{2} \mathcal{F}_{\sigma, \lambda}^{g}\left(\eta_{2}-\eta_{1}\right), \eta_{3}+\frac{\check{q}_{2}^{j}}{2} \mathcal{F}_{\sigma, \lambda}^{g}\left(\eta_{4}-\eta_{3}\right)\right), \\
& \frac{\check{q}_{1} \check{q}_{2}}{\mathcal{F}_{\sigma, \lambda}^{g}\left(\eta_{4}-\eta_{3}\right) \mathcal{F}_{\sigma, \lambda}^{g}\left(\eta_{2}-\eta_{1}\right)} \sum_{k=0}^{\infty} \sum_{j=0}^{\infty} \check{q}_{1}^{K} \check{q}_{2}^{j} \mathcal{G}\left(\eta_{1}+\frac{\check{q}_{1}^{K}}{2} \mathcal{F}_{\sigma, \lambda}^{g}\left(\eta_{2}-\eta_{1}\right), \eta_{3}+\frac{\check{q}_{2}^{j}}{2} \mathcal{F}_{\sigma, \lambda}^{g}\left(\eta_{4}-\eta_{3}\right)\right) \\
& -\frac{1}{6 \mathcal{F}_{\sigma, \lambda}^{g}\left(\eta_{2}-\eta_{1}\right) \mathcal{F}_{\sigma, \lambda}^{g}\left(\eta_{4}-\eta_{3}\right)} \sum_{\kappa=1}^{\infty} \sum_{j=1}^{\infty} \check{q}_{1}^{\kappa} \mathcal{G}\left(\eta_{1}+\frac{\check{q}_{1}^{K}}{2} \mathcal{F}_{\sigma, \lambda}^{g}\left(\eta_{2}-\eta_{1}\right), \eta_{3}+\frac{\check{q}_{2}^{j}}{2} \mathcal{F}_{\sigma, \lambda}^{g}\left(\eta_{4}-\eta_{3}\right)\right) \\
& =\frac{1}{6 \mathcal{F}_{\sigma, \lambda}^{9}\left(\eta_{2}-\eta_{1}\right) \mathcal{F}_{\sigma, \lambda}^{9}\left(\eta_{4}-\eta_{3}\right)} \mathcal{G}\left(\frac{2 \eta_{1}+\mathcal{F}_{\sigma, \lambda}^{9}\left(\eta_{2}-\eta_{1}\right)}{2}, \eta_{3}\right) \\
& -\frac{1}{6 \mathcal{F}_{\sigma, \lambda}^{9}\left(\eta_{2}-\eta_{1}\right) \mathcal{F}_{\sigma, \lambda}^{9}\left(\eta_{4}-\eta_{3}\right)} \sum_{\kappa=0}^{\infty} \check{q}_{1}^{\kappa} \mathcal{G}\left(\eta_{1}+\frac{\check{q}_{1}^{K}}{2} \mathcal{F}_{\sigma, \lambda}^{g}\left(\eta_{2}-\eta_{1}\right), \eta_{3}\right) \\
& -\frac{1}{6 \mathcal{F}_{\sigma, \lambda}^{9}\left(\eta_{2}-\eta_{1}\right) \mathcal{F}_{\sigma, \lambda}^{9}\left(\eta_{4}-\eta_{3}\right)} \sum_{k=1}^{\infty} \sum_{j=1}^{\infty} \check{q}_{1}^{\kappa} \mathcal{G}\left(\eta_{1}+\frac{\check{q}_{1}^{k}}{2} \mathcal{F}_{\sigma, \lambda}^{g}\left(\eta_{2}-\eta_{1}\right), \eta_{3}+\frac{\check{q}_{2}^{j}}{2} \mathcal{F}_{\sigma, \lambda}^{g}\left(\eta_{4}-\eta_{3}\right)\right) \text {, } \\
& \frac{1}{6 \mathcal{F}_{\sigma, \lambda}^{g}\left(\eta_{2}-\eta_{1}\right) \mathcal{F}_{\sigma, \lambda}^{g}\left(\eta_{4}-\eta_{3}\right)} \sum_{\kappa=1}^{\infty} \sum_{j=0}^{\infty} \check{q}_{1}^{\kappa} \mathcal{G}\left(\eta_{1}+\frac{\check{q}_{1}^{K}}{2} \mathcal{F}_{\sigma, \lambda}^{g}\left(\eta_{2}-\eta_{1}\right), \eta_{3}+\frac{\check{q}_{2}^{j}}{2} \mathcal{F}_{\sigma, \lambda}^{g}\left(\eta_{4}-\eta_{3}\right)\right) \\
& =-\frac{1}{6 \mathcal{F}_{\sigma, \lambda}^{9}\left(\eta_{2}-\eta_{1}\right) \mathcal{F}_{\sigma, \lambda}^{9}\left(\eta_{4}-\eta_{3}\right)} \mathcal{G}\left(\frac{2 \eta_{1}+\mathcal{F}_{\sigma, \lambda}^{9}\left(\eta_{2}-\eta_{1}\right)}{2}, \frac{2 \eta_{3}+\mathcal{F}_{\sigma, \lambda}^{9}\left(\eta_{4}-\eta_{3}\right)}{2}\right) \\
& +\frac{1}{6 \mathcal{F}_{\sigma, \lambda}^{9}\left(\eta_{2}-\eta_{1}\right)\left(\eta_{4}-\eta_{3}\right)} \sum_{\kappa=0}^{\infty} \check{q}_{1}^{\kappa} \mathcal{G}\left(\eta_{1}+\frac{\check{q}_{1}^{K}}{2} \mathcal{F}_{\sigma, \lambda}^{9}\left(\eta_{2}-\eta_{1}\right), \frac{2 \eta_{3}+\mathcal{F}_{\sigma, \lambda}^{g}\left(\eta_{4}-\eta_{3}\right)}{2}\right) \\
& -\frac{\check{q}_{2}}{\mathcal{F}_{\sigma, \lambda}^{g}\left(\eta_{2}-\eta_{1}\right) \mathcal{F}_{\sigma, \lambda}^{g}\left(\eta_{4}-\eta_{3}\right)} \sum_{k=0}^{\infty} \sum_{j=0}^{\infty} \check{q}_{1}^{\kappa} \check{q}_{2}^{j} \mathcal{G}\left(\eta_{1}+\frac{\check{q}_{1}^{k}}{2} \mathcal{F}_{\sigma, \lambda}^{g}\left(\eta_{2}-\eta_{1}\right), \eta_{3}+\frac{\check{q}_{2}^{j}}{2} \mathcal{F}_{\sigma, \lambda}^{g}\left(\eta_{4}-\eta_{3}\right)\right), \\
& +\frac{1}{6 \mathcal{F}_{\sigma, \lambda}^{g}\left(\eta_{2}-\eta_{1}\right) \mathcal{F}_{\sigma, \lambda}^{g}\left(\eta_{4}-\eta_{3}\right)} \sum_{\kappa=1}^{\infty} \sum_{j=1}^{\infty} \check{q}_{1}^{\kappa} \mathcal{G}\left(\eta_{1}+\frac{\check{q}_{1}^{k}}{2} \mathcal{F}_{\sigma, \lambda}^{g}\left(\eta_{2}-\eta_{1}\right), \eta_{3}+\frac{\check{q}_{2}^{j}}{2} \mathcal{F}_{\sigma, \lambda}^{g}\left(\eta_{4}-\eta_{3}\right)\right), \\
& \frac{\check{q}_{1}}{6 \mathcal{F}_{\sigma, \lambda}^{g}\left(\eta_{2}-\eta_{1}\right) \mathcal{F}_{\sigma, \lambda}^{g}\left(\eta_{4}-\eta_{3}\right)} \sum_{k=0}^{\infty} \sum_{j=1}^{\infty} \check{q}_{1}^{\kappa} \mathcal{G}\left(\eta_{1}+\frac{\check{q}_{1}^{K}}{2} \mathcal{F}_{\sigma, \lambda}^{g}\left(\eta_{2}-\eta_{1}\right), \eta_{3}+\frac{\check{q}_{2}^{j}}{2} \mathcal{F}_{\sigma, \lambda}^{g}\left(\eta_{4}-\eta_{3}\right)\right) \\
& =\frac{\check{q}_{1}}{6 \mathcal{F}_{\sigma, \lambda}^{g}\left(\eta_{2}-\eta_{1}\right) \mathcal{F}_{\sigma, \lambda}^{9}\left(\eta_{4}-\eta_{3}\right)}\left[-\sum_{k=0}^{\infty} \check{q}_{1}^{K} \mathcal{G}\left(\eta_{1}+\frac{\check{q}_{1}^{K}}{2} \mathcal{F}_{\sigma, \lambda}^{g}\left(\eta_{2}-\eta_{1}\right), \frac{2 \eta_{3}+\mathcal{F}_{\sigma, \lambda}^{g}\left(\eta_{4}-\eta_{3}\right)}{2}\right)\right. \\
& \left.+\sum_{\kappa=0}^{\infty} \check{q}_{1}^{K} \mathcal{G}\left(\eta_{1}+\frac{\check{q}_{1}^{K}}{2} \mathcal{F}_{\sigma, \lambda}^{\vartheta}\left(\eta_{2}-\eta_{1}\right), \eta_{3}\right)\right] \\
& -\frac{\check{q}_{1}}{6 \mathcal{F}_{\sigma, \lambda}^{g}\left(\eta_{2}-\eta_{1}\right) \mathcal{F}_{\sigma, \lambda}^{g}\left(\eta_{4}-\eta_{3}\right)} \sum_{k=0}^{\infty} \sum_{j=0}^{\infty} \check{q}_{1}^{K} \mathcal{G}\left(\eta_{1}+\frac{\check{q}_{1}^{K}}{2} \mathcal{F}_{\sigma, \lambda}^{g}\left(\eta_{2}-\eta_{1}\right), \eta_{3}+\frac{\check{q}_{2}^{j}}{2} \mathcal{F}_{\sigma, \lambda}^{g}\left(\eta_{4}-\eta_{3}\right)\right), \\
& -\frac{1}{6 \mathcal{F}_{\sigma, \lambda}^{g}\left(\eta_{2}-\eta_{1}\right) \mathcal{F}_{\sigma, \lambda}^{9}\left(\eta_{4}-\eta_{3}\right)} \sum_{k=1}^{\infty} \sum_{j=1}^{\infty} \check{q}_{2}^{j} \mathcal{G}\left(\eta_{1}+\frac{\check{q}_{1}^{k}}{2} \mathcal{F}_{\sigma, \lambda}^{g}\left(\eta_{2}-\eta_{1}\right), \eta_{3}+\frac{\check{q}_{2}^{j}}{2} \mathcal{F}_{\sigma, \lambda}^{g}\left(\eta_{4}-\eta_{3}\right)\right) \\
& =\frac{1}{6 \mathcal{F}_{\sigma, \lambda}^{9}\left(\eta_{2}-\eta_{1}\right) \mathcal{F}_{\sigma, \lambda}^{9}\left(\eta_{4}-\eta_{3}\right)} \mathcal{G}\left(\eta_{1}, \frac{2 \eta_{3}+\mathcal{F}_{\sigma, \lambda}^{9}\left(\eta_{4}-\eta_{3}\right)}{2}\right) \\
& -\frac{1}{6 \mathcal{F}_{\sigma, \lambda}^{g}\left(\eta_{2}-\eta_{1}\right) \mathcal{F}_{\sigma, \lambda}^{g}\left(\eta_{4}-\eta_{3}\right)} \sum_{j=0}^{\infty} \check{q}_{2}^{j} \mathcal{G}\left(\eta_{1}, \eta_{3}+\frac{\check{q}_{2}^{j}}{2} \mathcal{F}_{\sigma, \lambda}^{g}\left(\eta_{4}-\eta_{3}\right)\right) \\
& -\frac{1}{6 \mathcal{F}_{\sigma, \lambda}^{g}\left(\eta_{2}-\eta_{1}\right) \mathcal{F}_{\sigma, \lambda}^{g}\left(\eta_{4}-\eta_{3}\right)} \sum_{k=1 j=1}^{\infty} \sum_{j=1}^{\infty} \check{q}_{2}^{j} \mathcal{G}\left(\eta_{1}+\frac{\check{q}_{1}^{k}}{2} \mathcal{F}_{\sigma, \lambda}^{g}\left(\eta_{2}-\eta_{1}\right), \eta_{3}+\frac{\check{q}_{2}^{j}}{2} \mathcal{F}_{\sigma, \lambda}^{g}\left(\eta_{4}-\eta_{3}\right)\right),
\end{aligned}
$$




$$
\begin{aligned}
& \frac{\check{q}_{2}}{6 \mathcal{F}_{\sigma, \lambda}^{9}\left(\eta_{2}-\eta_{1}\right) \mathcal{F}_{\sigma, \lambda}^{g}\left(\eta_{4}-\eta_{3}\right)} \sum_{\kappa=1}^{\infty} \sum_{j=0}^{\infty} \check{q}_{2}^{j} \mathcal{G}\left(\eta_{1}+\frac{\check{q}_{1}^{\kappa}}{2} \mathcal{F}_{\sigma, \lambda}^{g}\left(\eta_{2}-\eta_{1}\right), \eta_{3}+\frac{\check{q}_{2}^{j}}{2} \mathcal{F}_{\sigma, \lambda}^{g}\left(\eta_{4}-\eta_{3}\right)\right) \\
& =-\frac{\check{q}_{2}}{6 \mathcal{F}_{\sigma, \lambda}^{9}\left(\eta_{2}-\eta_{1}\right)_{\mathcal{F}_{\sigma, \lambda}}^{g}\left(\eta_{4}-\eta_{3}\right)}\left[-\sum_{j=0}^{\infty} \check{q}_{2}^{j} \mathcal{G}\left(\frac{2 \eta_{1}+\mathcal{F}_{\sigma, \lambda}^{9}\left(\eta_{2}-\eta_{1}\right)}{2}, \eta_{3}+\frac{\check{q}_{2}^{j}}{2} \mathcal{F}_{\sigma, \lambda}^{9}\left(\eta_{4}-\eta_{3}\right)\right)\right. \\
& \left.+\sum_{j=0}^{\infty} \check{q}_{2}^{j} \mathcal{G}\left(\eta_{1}, \eta_{3}+\frac{\check{q}_{2}^{j}}{2} \mathcal{F}_{\sigma, \lambda}^{g}\left(\eta_{4}-\eta_{3}\right)\right)\right] \\
& +\frac{\check{q}_{2}}{6 \mathcal{F}_{\sigma, \lambda}^{9}\left(\eta_{2}-\eta_{1}\right) \mathcal{F}_{\sigma, \lambda}^{g}\left(\eta_{4}-\eta_{3}\right)} \sum_{k=0}^{\infty} \sum_{j=0}^{\infty} \check{q}_{2}^{j} \mathcal{G}\left(\eta_{1}+\frac{\check{q}_{1}^{K}}{2} \mathcal{F}_{\sigma, \lambda}^{g}\left(\eta_{2}-\eta_{1}\right), \eta_{3}+\frac{\check{q}_{2}^{j}}{2} \mathcal{F}_{\sigma, \lambda}^{g}\left(\eta_{4}-\eta_{3}\right)\right), \\
& \frac{1}{6 \mathcal{F}_{\sigma, \lambda}^{g}\left(\eta_{2}-\eta_{1}\right) \mathcal{F}_{\sigma, \lambda}^{g}\left(\eta_{4}-\eta_{3}\right)} \sum_{\kappa=0}^{\infty} \sum_{j=1}^{\infty} \check{q}_{2}^{j} \mathcal{G}\left(\eta_{1}+\frac{\check{q}_{1}^{\kappa}}{2} \mathcal{F}_{\sigma, \lambda}^{g}\left(\eta_{2}-\eta_{1}\right), \eta_{3}+\frac{\check{q}_{2}^{j}}{2} \mathcal{F}_{\sigma, \lambda}^{g}\left(\eta_{4}-\eta_{3}\right)\right) \\
& =-\frac{1}{6 \mathcal{F}_{\sigma, \lambda}^{9}\left(\eta_{2}-\eta_{1}\right) \mathcal{F}_{\sigma, \lambda}^{9}\left(\eta_{4}-\eta_{3}\right)} \mathcal{G}\left(\frac{2 \eta_{1}+\mathcal{F}_{\sigma, \lambda}^{9}\left(\eta_{2}-\eta_{1}\right)}{2}, \frac{2 \eta_{3}+\mathcal{F}_{\sigma, \lambda}^{9}\left(\eta_{4}-\eta_{3}\right)}{2}\right) \\
& +\frac{1}{6 \mathcal{F}_{\sigma, \lambda}^{g}\left(\eta_{2}-\eta_{1}\right) \mathcal{F}_{\sigma, \lambda}^{9}\left(\eta_{4}-\eta_{3}\right)} \sum_{j=0}^{\infty} \check{q}_{2}^{j} \mathcal{G}\left(\frac{2 \eta_{1}+\mathcal{F}_{\sigma, \lambda}^{9}\left(\eta_{2}-\eta_{1}\right)}{2}, \eta_{3}+\frac{\check{q}_{2}^{j}}{2} \mathcal{F}_{\sigma, \lambda}^{g}\left(\eta_{4}-\eta_{3}\right)\right) \\
& +\frac{1}{6 \mathcal{F}_{\sigma, \lambda}^{g}\left(\eta_{2}-\eta_{1}\right) \mathcal{F}_{\sigma, \lambda}^{g}\left(\eta_{4}-\eta_{3}\right)} \sum_{\kappa=1}^{\infty} \sum_{j=1}^{\infty} \check{q}_{2}^{j} \mathcal{G}\left(\eta_{1}+\frac{\check{q}_{1}^{\kappa}}{2} \mathcal{F}_{\sigma, \lambda}^{g}\left(\eta_{2}-\eta_{1}\right), \eta_{3}+\frac{\check{q}_{2}^{j}}{2} \mathcal{F}_{\sigma, \lambda}^{g}\left(\eta_{4}-\eta_{3}\right)\right), \\
& -\frac{\check{q}_{2}}{6 \mathcal{F}_{\sigma, \lambda}^{\theta}\left(\eta_{2}-\eta_{1}\right) \mathcal{F}_{\sigma, \lambda}^{g}\left(\eta_{4}-\eta_{3}\right)} \sum_{k=0}^{\infty} \sum_{j=0}^{\infty} \check{q}_{2}^{j} \mathcal{G}\left(\eta_{1}+\frac{\check{q}_{1}^{k}}{2} \mathcal{F}_{\sigma, \lambda}^{\vartheta}\left(\eta_{2}-\eta_{1}\right), \eta_{3}+\frac{\check{q}_{2}^{j}}{2} \mathcal{F}_{\sigma, \lambda}^{\vartheta}\left(\eta_{4}-\eta_{3}\right)\right) \\
& \times \frac{1}{36 \mathcal{F}_{\sigma, \lambda}^{g}\left(\eta_{2}-\eta_{1}\right) \mathcal{F}_{\sigma, \lambda}^{g}\left(\eta_{4}-\eta_{3}\right)} \sum_{\kappa=1}^{\infty} \sum_{j=1}^{\infty} \mathcal{G}\left(\eta_{1}+\frac{\check{q}_{1}^{K}}{2} \mathcal{F}_{\sigma, \lambda}^{g}\left(\eta_{2}-\eta_{1}\right), \eta_{3}+\frac{\check{q}_{2}^{j}}{2} \mathcal{F}_{\sigma, \lambda}^{g}\left(\eta_{4}-\eta_{3}\right)\right) \\
& =\frac{\mathcal{G}\left(\eta_{1}, \eta_{3}\right)}{36 \mathcal{F}_{\sigma, \lambda}^{g}\left(\eta_{2}-\eta_{1}\right) \mathcal{F}_{\sigma, \lambda}^{9}\left(\eta_{4}-\eta_{3}\right)} \\
& +\frac{1}{36 \mathcal{F}_{\sigma, \lambda}^{9}\left(\eta_{2}-\eta_{1}\right) \mathcal{F}_{\sigma, \lambda}^{g}\left(\eta_{4}-\eta_{3}\right)} \sum_{\kappa=1}^{\infty} \sum_{j=1}^{\infty} \mathcal{G}\left(\eta_{1}+\frac{\check{q}_{1}^{K}}{2} \mathcal{F}_{\sigma, \lambda}^{g}\left(\eta_{2}-\eta_{1}\right), \eta_{3}+\frac{\check{q}_{2}^{j}}{2} \mathcal{F}_{\sigma, \lambda}^{g}\left(\eta_{4}-\eta_{3}\right)\right), \\
& -\frac{1}{36 \mathcal{F}_{\sigma, \lambda}^{g}\left(\eta_{2}-\eta_{1}\right) \mathcal{F}_{\sigma, \lambda}^{g}\left(\eta_{4}-\eta_{3}\right)} \sum_{k=1}^{\infty} \sum_{j=0}^{\infty} \mathcal{G}\left(\eta_{1}+\frac{\check{q}_{1}^{\kappa}}{2} \mathcal{F}_{\sigma, \lambda}^{g}\left(\eta_{2}-\eta_{1}\right), \eta_{3}+\frac{\check{q}_{2}^{j}}{2} \mathcal{F}_{\sigma, \lambda}^{g}\left(\eta_{4}-\eta_{3}\right)\right) \\
& =-\frac{1}{36 \mathcal{F}_{\sigma, \lambda}^{g}\left(\eta_{2}-\eta_{1}\right) \mathcal{F}_{\sigma, \lambda}^{g}\left(\eta_{4}-\eta_{3}\right)} \mathcal{G}\left(\eta_{1}, \frac{2 \eta_{3}+\mathcal{F}_{\sigma, \lambda}^{9}\left(\eta_{4}-\eta_{3}\right)}{2}\right) \\
& -\frac{1}{36 \mathcal{F}_{\sigma, \lambda}^{g}\left(\eta_{2}-\eta_{1}\right) \mathcal{F}_{\sigma, \lambda}^{g}\left(\eta_{4}-\eta_{3}\right)} \sum_{k=1}^{\infty} \sum_{j=1}^{\infty} \mathcal{G}\left(\eta_{1}+\frac{\check{q}_{1}^{K}}{2} \mathcal{F}_{\sigma, \lambda}^{g}\left(\eta_{2}-\eta_{1}\right), \eta_{3}+\frac{\check{q}_{2}^{j}}{2} \mathcal{F}_{\sigma, \lambda}^{g}\left(\eta_{4}-\eta_{3}\right)\right), \\
& -\frac{1}{36 \mathcal{F}_{\sigma, \lambda}^{g}\left(\eta_{2}-\eta_{1}\right) \mathcal{F}_{\sigma, \lambda}^{g}\left(\eta_{4}-\eta_{3}\right)} \sum_{k=0}^{\infty} \sum_{j=1}^{\infty} \mathcal{G}\left(\eta_{1}+\frac{\check{q}_{1}^{k}}{2} \mathcal{F}_{\sigma, \lambda}^{g}\left(\eta_{2}-\eta_{1}\right), \eta_{3}+\frac{\check{q}_{2}^{j}}{2} \mathcal{F}_{\sigma, \lambda}^{g}\left(\eta_{4}-\eta_{3}\right)\right) \\
& =-\frac{1}{36 \mathcal{F}_{\sigma, \lambda}^{9}\left(\eta_{2}-\eta_{1}\right) \mathcal{F}_{\sigma, \lambda}^{g}\left(\eta_{4}-\eta_{3}\right)} \mathcal{G}\left(\frac{2 \eta_{1}+\mathcal{F}_{\sigma, \lambda}^{9}\left(\eta_{2}-\eta_{1}\right)}{2}, \eta_{3}\right) \\
& -\frac{1}{36 \mathcal{F}_{\sigma, \lambda}^{g}\left(\eta_{2}-\eta_{1}\right) \mathcal{F}_{\sigma, \lambda}^{g}\left(\eta_{4}-\eta_{3}\right)} \sum_{\kappa=1}^{\infty} \sum_{j=1}^{\infty} \mathcal{G}\left(\eta_{1}+\frac{\check{q}_{1}^{K}}{2} \mathcal{F}_{\sigma, \lambda}^{\vartheta}\left(\eta_{2}-\eta_{1}\right), \eta_{3}+\frac{\check{q}_{2}^{j}}{2} \mathcal{F}_{\sigma, \lambda}^{g}\left(\eta_{4}-\eta_{3}\right)\right), \\
& \frac{1}{36 \mathcal{F}_{\sigma, \lambda}^{g}\left(\eta_{2}-\eta_{1}\right) \mathcal{F}_{\sigma, \lambda}^{g}\left(\eta_{4}-\eta_{3}\right)} \sum_{k=0}^{\infty} \sum_{j=0}^{\infty} \mathcal{G}\left(\eta_{1}+\frac{\check{q}_{1}^{K}}{2} \mathcal{F}_{\sigma, \lambda}^{g}\left(\eta_{2}-\eta_{1}\right), \eta_{3}+\frac{\check{q}_{2}^{j}}{2} \mathcal{F}_{\sigma, \lambda}^{g}\left(\eta_{4}-\eta_{3}\right)\right)
\end{aligned}
$$




$$
\begin{aligned}
= & \frac{1}{36 \mathcal{F}_{\sigma, \lambda}^{9}\left(\eta_{2}-\eta_{1}\right) \mathcal{F}_{\sigma, \lambda}^{\theta}\left(\eta_{4}-\eta_{3}\right)} \mathcal{G}\left(\frac{2 \eta_{1}+\mathcal{F}_{\sigma, \lambda}^{\vartheta}\left(\eta_{2}-\eta_{1}\right)}{2}, \frac{2 \eta_{3}+\mathcal{F}_{\sigma, \lambda}^{\vartheta}\left(\eta_{4}-\eta_{3}\right)}{2}\right) \\
& +\frac{1}{36 \mathcal{F}_{\sigma, \lambda}^{\vartheta}\left(\eta_{2}-\eta_{1}\right) \mathcal{F}_{\sigma, \lambda}^{\vartheta}\left(\eta_{4}-\eta_{3}\right)} \sum_{\kappa=1}^{\infty} \sum_{j=1}^{\infty} \mathcal{G}\left(\eta_{1}+\frac{\check{q}_{1}^{\kappa}}{2} \mathcal{F}_{\sigma, \lambda}^{\vartheta}\left(\eta_{2}-\eta_{1}\right), \eta_{3}+\frac{\check{q}_{2}^{j}}{2} \mathcal{F}_{\sigma, \lambda}^{\vartheta}\left(\eta_{4}-\eta_{3}\right)\right) .
\end{aligned}
$$

Analogously, adopting the same technique we can adding all of the above computed $q_{1} q_{2}$-integrals, we obtain the remaining three $q_{1} q_{2}$-integrals, and later on, acquire the following identity:

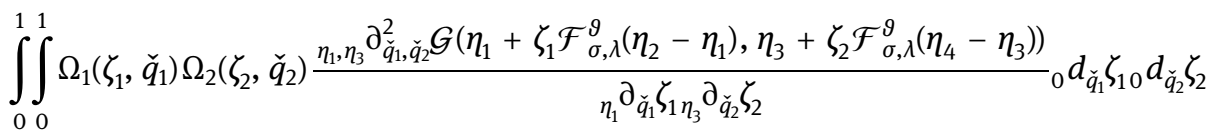

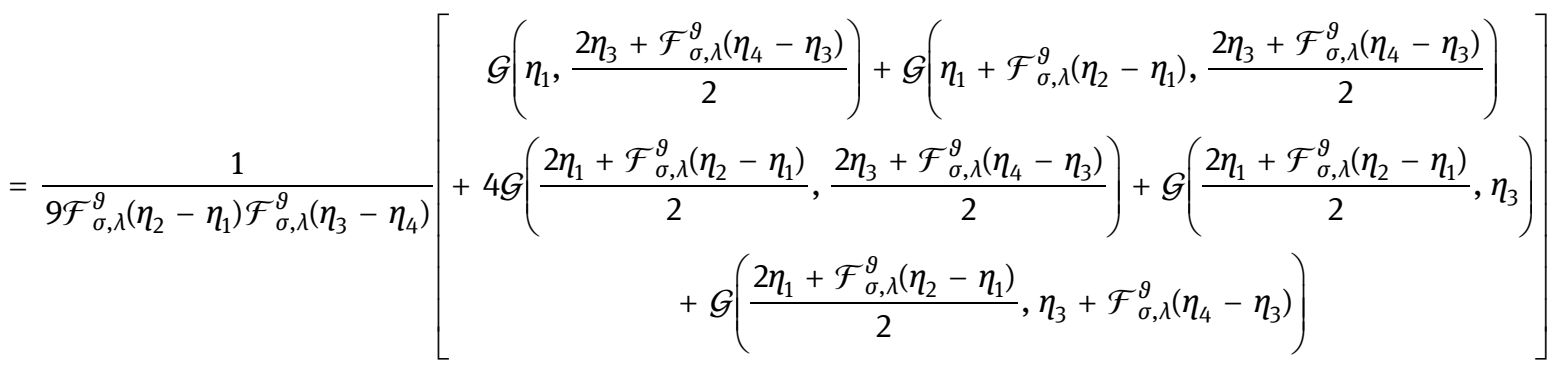

$$
\begin{aligned}
& +\frac{1}{36 \mathcal{F}_{\sigma, \lambda}^{\vartheta}\left(\eta_{2}-\eta_{1}\right) \mathcal{F}_{\sigma, \lambda}^{\vartheta}\left(\eta_{3}-\eta_{4}\right)}\left[\begin{array}{c}
\mathcal{G}\left(\eta_{1}, \eta_{3}\right)+\mathcal{G}\left(\eta_{1}+\mathcal{F}_{\sigma, \lambda}^{\vartheta}\left(\eta_{2}-\eta_{1}\right), \eta_{3}\right)+\mathcal{G}\left(\eta_{1}, \eta_{3}+\mathcal{F}_{\sigma, \lambda}^{\vartheta}\left(\eta_{4}-\eta_{3}\right)\right) \\
+\mathcal{G}\left(\eta_{1}+\mathcal{F}_{\sigma, \lambda}^{\vartheta}\left(\eta_{2}-\eta_{1}\right), \eta_{3}+\mathcal{F}_{\sigma, \lambda}^{\vartheta}\left(\eta_{4}-\eta_{3}\right)\right)
\end{array}\right] \\
& \left.\left.-\frac{1-\check{q}_{1}}{6 \mathcal{F}_{\sigma, \lambda}^{9}\left(\eta_{2}-\eta_{1}\right) \mathcal{F}_{\sigma, \lambda}^{\vartheta}\left(\eta_{3}-\eta_{4}\right)}\right]+4 \sum_{\kappa=0}^{\infty} \check{q}_{1}^{\kappa} \mathcal{G}\left(\eta_{1}+\check{q}_{1}^{\kappa} \mathcal{F}_{\sigma, \lambda}^{\vartheta}\left(\eta_{2}-\eta_{1}\right), \frac{2 \eta_{3}+\mathcal{F}_{\sigma, \lambda}^{\vartheta}\left(\eta_{4}-\eta_{3}\right)}{2}\right)\right] \\
& +\sum_{\kappa=0}^{\infty} \check{q}_{1}^{\kappa} \mathcal{G}\left(\eta_{1}+\check{q}_{1}^{\kappa} \mathcal{F}_{\sigma, \lambda}^{\vartheta}\left(\eta_{2}-\eta_{1}\right), \eta_{3}+\mathcal{F}_{\sigma, \lambda}^{\vartheta}\left(\eta_{4}-\eta_{3}\right)\right)
\end{aligned}
$$

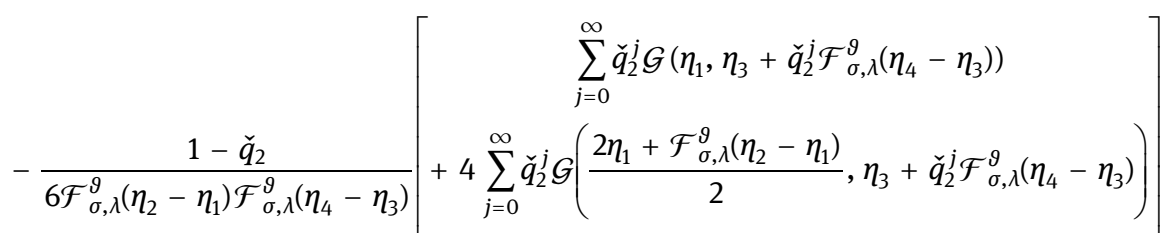

$$
\begin{aligned}
& +\sum_{j=0}^{\infty} \check{q}_{2}^{j} \mathcal{G}\left(\eta_{2}, \eta_{3}+\check{q}_{2}^{j} \mathcal{F}_{\sigma, \lambda}^{\vartheta}\left(\eta_{4}-\eta_{3}\right)\right) \\
& +\frac{\left(1-\check{q}_{1}\right)\left(1-\check{q}_{2}\right)}{\mathcal{F}_{\sigma, \lambda}^{g}\left(\eta_{2}-\eta_{1}\right) \mathcal{F}_{\sigma, \lambda}^{g}\left(\eta_{4}-\eta_{3}\right)} \sum_{\kappa=0}^{\infty} \sum_{j=0}^{\infty}\left[\mathcal{G}\left(\eta_{1}+\check{q}_{1}^{\kappa} \mathcal{F}_{\sigma, \lambda}^{g}\left(\eta_{2}-\eta_{1}\right), \eta_{3}+\check{q}_{2}^{j} \mathcal{F}_{\sigma, \lambda}^{g}\left(\eta_{4}-\eta_{3}\right)\right)\right] \text {. }
\end{aligned}
$$

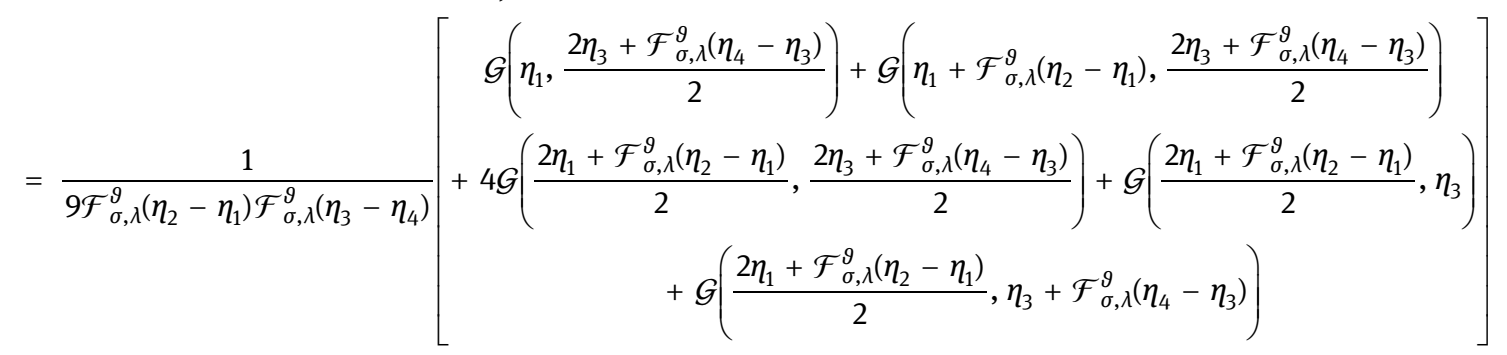




$$
\begin{aligned}
& +\frac{1}{36 \mathcal{F}_{\sigma, \lambda}^{9}\left(\eta_{2}-\eta_{1}\right) \mathcal{F}_{\sigma, \lambda}^{9}\left(\eta_{3}-\eta_{4}\right)}\left[\begin{array}{c}
\mathcal{G}\left(\eta_{1}, \eta_{3}\right)+\mathcal{G}\left(\eta_{1}+\mathcal{F}_{\sigma, \lambda}^{9}\left(\eta_{2}-\eta_{1}\right), \eta_{3}\right)+\mathcal{G}\left(\eta_{1}, \eta_{3}+\mathcal{F}_{\sigma, \lambda}^{9}\left(\eta_{4}-\eta_{3}\right)\right) \\
+\mathcal{G}\left(\eta_{1}+\mathcal{F}_{\sigma, \lambda}^{g}\left(\eta_{2}-\eta_{1}\right), \eta_{3}+\mathcal{F}_{\sigma, \lambda}^{9}\left(\eta_{4}-\eta_{3}\right)\right)
\end{array}\right] \\
& -\frac{1}{6\left(\mathcal{F}_{\sigma, \lambda}^{9}\left(\eta_{2}-\eta_{1}\right)\right)^{2} \mathcal{F}_{\sigma, \lambda}^{9}\left(\eta_{4}-\eta_{3}\right)} \int_{\eta_{1}}^{\eta_{1}+\mathcal{F}_{\sigma, \lambda}^{9}\left(\eta_{2}-\eta_{1}\right)}\left[\mathcal{G}\left(x, \eta_{3}\right)+4 \mathcal{G}\left(x, \frac{2 \eta_{3}+\mathcal{F}_{\sigma, \lambda}^{9}\left(\eta_{4}-\eta_{3}\right)}{2}\right)+\mathcal{G}\left(x, \eta_{3}\right)\right]{ }_{0} d_{\check{q}_{1}} x \\
& -\frac{1}{6 \mathcal{F}_{\sigma, \lambda}^{9}\left(\eta_{2}-\eta_{1}\right)\left(\mathcal{F}_{\sigma, \lambda}^{9}\left(\eta_{4}-\eta_{3}\right)\right)^{2}} \int_{\eta_{3}}^{\eta_{3}+\mathcal{F}_{\sigma, \lambda}^{9}\left(\eta_{4}-\eta_{3}\right)}\left[\mathcal{G}\left(\eta_{1}, y\right)+4 \mathcal{G}\left(\frac{2 \eta_{1}+\mathcal{F}_{\sigma, \lambda}^{9}\left(\eta_{2}-\eta_{1}\right)}{2}, y\right)+\mathcal{G}\left(\eta_{2}, y\right)\right]_{0} d_{\check{q}_{1}} y \\
& +\frac{1}{\mathcal{F}_{\sigma, \lambda}^{9}\left(\eta_{2}-\eta_{1}\right)\left(\mathcal{F}_{\sigma, \lambda}^{9}\left(\eta_{4}-\eta_{3}\right)\right)^{2}} \int_{\eta_{1}}^{\eta_{1}+\mathcal{F}_{\sigma, \lambda}^{9}\left(\eta_{2}-\eta_{1}\right)} \int_{\eta_{3}}^{\eta_{3}+\mathcal{F}_{\sigma, \lambda}^{9}\left(\eta_{4}-\eta_{3}\right)} \mathcal{G}(x, y)_{0} d_{\check{q}_{1}} x_{0} d_{\check{q}_{1}} y .
\end{aligned}
$$

By multiplying both sides of (3.4) by $\mathcal{F}_{\sigma, \lambda}^{\vartheta}\left(\eta_{2}-\eta_{1}\right) \mathcal{F}_{\sigma, \lambda}^{\vartheta}\left(\eta_{4}-\eta_{3}\right)$, we get the desired result.

Remark 3.1. Lemma 5.1 leads to the conclusion as follows:

I. If $\mathcal{F}_{\sigma, \lambda}^{\vartheta}\left(\eta_{2}-\eta_{1}\right)=\eta_{2}-\eta_{1}$ and $\mathcal{F}_{\sigma, \lambda}^{9}\left(\eta_{4}-\eta_{3}\right)=\eta_{4}-\eta_{3}$, then we get Lemma 4 of ref. [35].

II. If $\check{q_{1}} \mapsto 1^{-}, \check{q_{2}} \mapsto 1^{-}, \mathcal{F}_{\sigma, \lambda}^{\vartheta}\left(\eta_{2}-\eta_{1}\right)=\eta_{2}-\eta_{1}$ and $\mathcal{F}_{\sigma, \lambda}^{\vartheta}\left(\eta_{4}-\eta_{3}\right)=\eta_{4}-\eta_{3}$, then we get Lemma 1 of ref. [41].

\section{New quantum estimates for generalized higher order strongly $\Psi$-convex functions}

According to the generalized concepts given in Section 2, our special attention is to deriving the two-variables $\check{q}_{1} \check{q}_{2}$-integral inequalities of Simpson-like type for two- variable generalized higher-order strongly $\Psi$-convex functions.

Theorem 4.1. Suppose that Assumptions (I) and (II) are

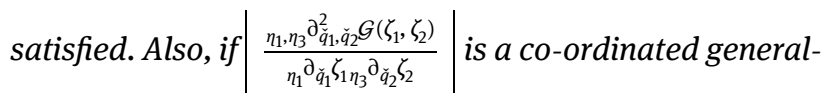
ized higher-order strongly $\Psi$-convex function, then

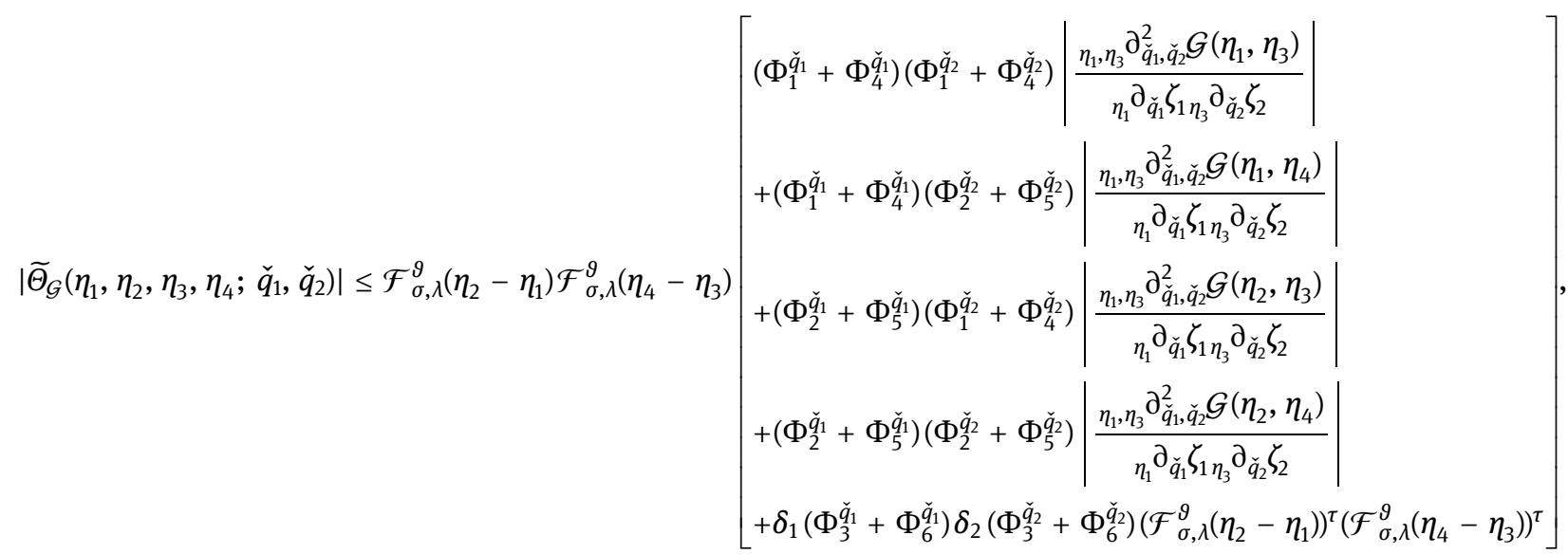

where

$$
\Phi_{1}^{\check{q}_{i}}=\left\{\begin{array}{ll}
\frac{1-4 \check{q}_{i}^{3}}{24\left(1+\check{q}_{i}\right)\left(1+\check{q}_{i}+\check{q}_{i}^{2}\right)}, & 0<\check{q}_{i}<\frac{1}{3}, 1 \leq i \leq 2, \\
\frac{1+12 \check{q}_{i}+12 \check{q}_{i}^{2}+36 \check{q}_{i}^{3}}{216\left(1+\check{q}_{i}\right)\left(1+\check{q}_{i}+\check{q}_{i}^{2}\right)}, & \frac{1}{3} \leq \check{q}_{i}<1,1 \leq i \leq 2,
\end{array} \quad \Phi_{2}^{\check{q}_{i}}= \begin{cases}\frac{1-2 \check{q}_{i}-2 \check{q}_{i}^{2}}{24\left(1+\check{q}_{i}\right)\left(1+\check{q}_{i}+\check{q}_{i}^{2}\right)}, \quad 0<\check{q}_{i}<\frac{1}{3}, 1 \leq i \leq 2, \\
\frac{18 \check{q}_{i}+18 \check{q}_{i}^{2}-7}{216\left(1+\check{q}_{i}\right)\left(1+\check{q}_{i}+\check{q}_{i}^{2}\right)}, \quad \frac{1}{3} \leq \check{q}_{i}<1,1 \leq i \leq 2,\end{cases}\right.
$$




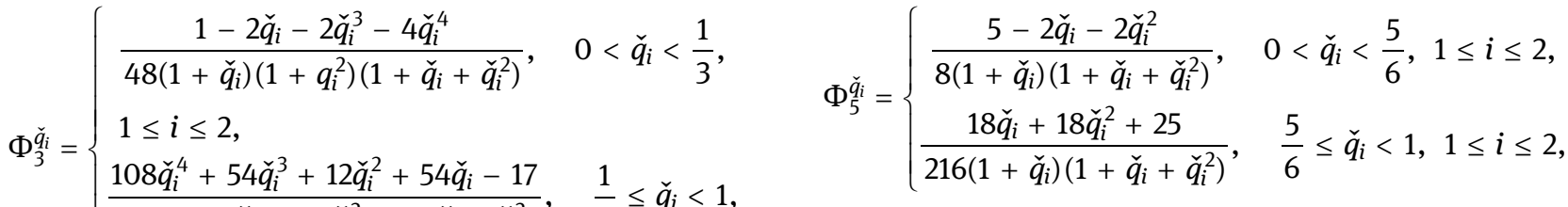

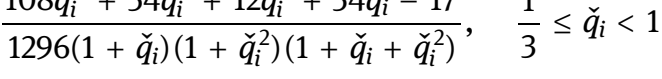

$$
\begin{aligned}
& 1 \leq i \leq 2 \text {, } \\
& \Phi_{4}^{\check{q}_{i}}= \begin{cases}\frac{-5+8 q+8 \check{q}_{i}^{2}-8 \check{q}_{i}^{3}}{24\left(1+\check{q}_{i}\right)\left(1+\check{q}_{i}+\check{q}_{i}^{2}\right)}, & 0<\check{q}_{i}<\frac{5}{6}, 1 \leq i \leq 2, \\
\frac{12 \check{q}_{i}+12 \check{q}_{i}^{2}+5}{216\left(1+\check{q}_{i}\right)\left(1+\check{q}_{i}+\check{q}_{i}^{2}\right)}, & \frac{5}{6} \leq \check{q}_{i}<1, \quad 1 \leq i \leq 2,\end{cases} \\
& \Phi_{6}^{\check{q}_{i}}=\left\{\begin{array}{l}
\frac{5-2 \check{q}_{i}+28 q_{k}^{2}-2 \check{q}_{i}^{3}-12 \check{q}_{i}^{4}}{48\left(1+\check{q}_{i}\right)\left(1+q_{i}^{2}\right)\left(1+\check{q}_{i}+\check{q}_{i}^{2}\right)}, \quad 0<\check{q}_{i}<\frac{5}{6}, \\
1 \leq i \leq 2, \\
\frac{108 \check{q}_{i}^{4}-54 \check{q}_{i}^{3}+96 \check{q}_{i}^{2}-54 \check{q}_{i}+115}{1296\left(1+\check{q}_{i}\right)\left(1+\check{q}_{i}^{2}\right)\left(1+\check{q}_{i}+\check{q}_{i}^{2}\right)}, \quad \frac{5}{6} \leq \check{q}_{i}<1, \\
1 \leq i \leq 2 .
\end{array}\right.
\end{aligned}
$$

Proof. By means of modulus property, the co-ordinated generalized higher-order strongly $\Psi$-convexity of

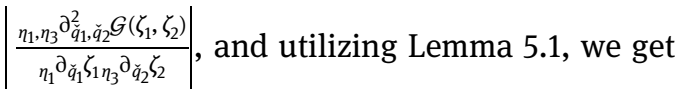

$\left|\widetilde{\Theta}_{\mathcal{G}}\left(\eta_{1}, \eta_{2}, \eta_{3}, \eta_{4} ; \check{q}_{1}, \check{q}_{2}\right)\right| \leq \mathcal{F}_{\sigma, \lambda}^{9}\left(\eta_{2}-\eta_{1}\right) \mathcal{F}_{\sigma, \lambda}^{9}\left(\eta_{4}-\eta_{3}\right)$

$$
\begin{aligned}
& \times \int_{0}^{1} \int_{0}^{1}\left|\Omega_{1}\left(\zeta_{1}, \check{q}_{1}\right) \Omega_{2}\left(w, \check{q}_{2}\right)\right|\left|\frac{\eta_{1}, \eta_{3} \partial_{\check{q}_{1}, \check{q}_{2}}^{2} \mathcal{G}\left(\eta_{1}+\zeta_{1} \mathcal{F}_{\sigma, \lambda}^{g}\left(\eta_{2}-\eta_{1}\right), \eta_{3}+w \mathcal{F}_{\sigma, \lambda}^{g}\left(\eta_{4}-\eta_{3}\right)\right)}{\eta_{1} \partial_{\check{q}_{1}} \zeta_{1 \eta_{3}} \partial_{\check{q}_{2}} \zeta_{2}}\right|{ }_{0} d_{\check{\check{l}}_{1}} \zeta_{\zeta_{10}} d_{\check{q}_{2}} w \\
& \leq \mathcal{F}_{\sigma, \lambda}^{\vartheta}\left(\eta_{2}-\eta_{1}\right) \mathcal{F}_{\sigma, \lambda}^{\vartheta}\left(\eta_{4}-\eta_{3}\right)
\end{aligned}
$$

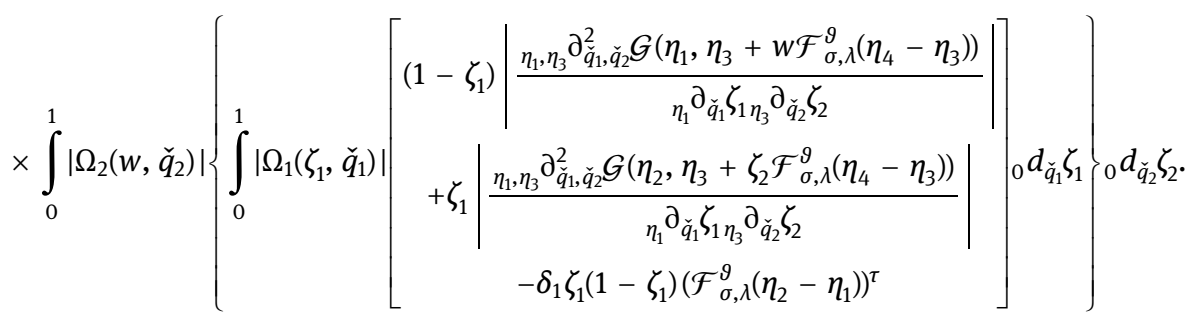

Simple computations of the $\check{q}_{1}$-integral mentioned in equation (4.1) yield

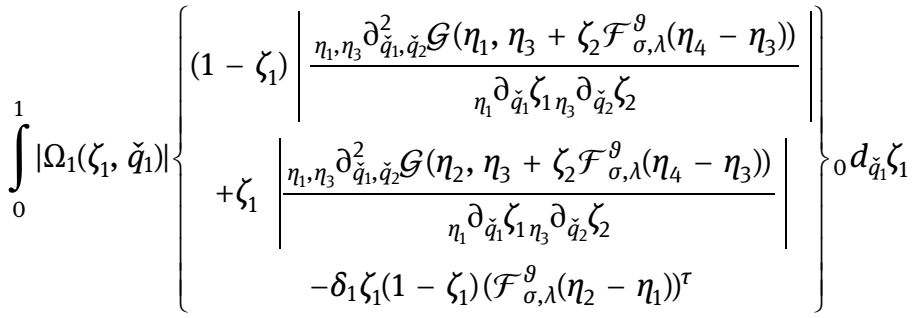

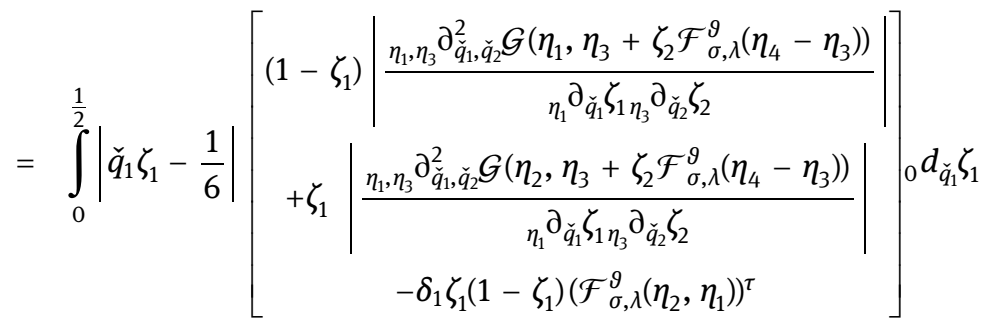

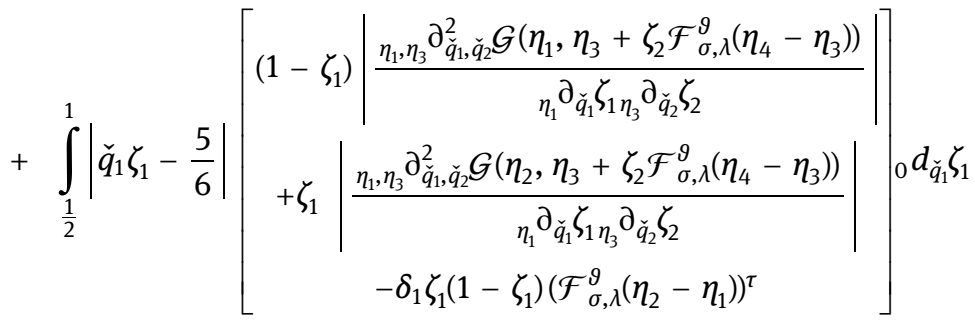


DE GRUYTER

Quantum estimates in two variable forms for Simpson-type inequalities

317

$$
\begin{aligned}
= & \left|\frac{\eta_{1}, \eta_{3} \partial_{\check{q}_{1}, \check{q}_{2}}^{2} \mathcal{G}\left(\eta_{1}, \eta_{3}+\zeta_{2} \eta_{2}\left(\eta_{4}, \eta_{3}\right)\right)}{\eta_{1} \partial_{\check{q}_{1}} \zeta_{1} \partial_{3} \partial_{\check{q}_{2}} \zeta_{2}}\right|\left[\int_{0}^{\frac{1}{2}}\left(1-\zeta_{1}\right)\left|\check{q}_{1} \zeta_{1}-\frac{1}{6}\right|{ }_{0} d_{\check{q}_{1}} \zeta_{1}+\int_{\frac{1}{2}}^{1}\left(1-\zeta_{1}\right)\left|\check{q}_{1} \zeta_{1}-\frac{5}{6}\right|{ }_{0} d_{\check{q}_{1}} \zeta_{1}\right] \\
& +\left|\frac{\eta_{1}, \eta_{3} \partial_{\check{q}_{1}, \check{q}_{2}}^{2} \mathcal{G}\left(\eta_{2}, \eta_{3}+\zeta_{2} \eta_{2}\left(\eta_{4}, \eta_{3}\right)\right)}{\eta_{1} \partial_{\check{q}_{1}} \zeta_{1} \eta_{3} \partial_{\check{q}_{2}} \zeta_{2}}\right|\left[\int_{0}^{\frac{1}{2}} \zeta_{1}\left|\check{q}_{1} \zeta_{1}-\frac{1}{6}\right|{ }_{0} d_{\check{q}_{1}} \zeta_{1}+\int_{\frac{1}{2}}^{1} \zeta_{1}\left|\check{q}_{1} \zeta_{1}-\frac{5}{6}\right|{ }_{0} d_{\check{q}_{1}} \zeta_{1}\right] \\
& -\delta_{1}\left(\mathcal{F}_{\sigma, \lambda}^{\vartheta}\left(\eta_{2}-\eta_{1}\right)\right)^{\tau}\left[\int_{0}^{\frac{1}{2}} \zeta_{1}\left(1-\zeta_{1}\right)\left|\check{q}_{1} \zeta_{1}-\frac{1}{6}\right|{ }_{0} d_{\check{q}_{1}} \zeta_{1}+\int_{\frac{1}{2}}^{1} \zeta_{1}\left(1-\zeta_{1}\right)\left|\check{q}_{1} \zeta_{1}-\frac{5}{6}\right|{ }_{0} d_{\check{q}_{1}} \zeta_{1}\right]
\end{aligned}
$$

Taking into account Definitions 2.11 and 2.12, we get

$$
\begin{aligned}
& \Phi_{1}^{\check{q}_{i}}=\int_{0}^{\frac{1}{2}}\left(1-\zeta_{1}\right)\left|\check{q}_{i} \zeta_{1}-\frac{1}{6}\right|{ }_{0} d_{\check{q}_{i}} \zeta_{1}= \begin{cases}\frac{1-4 \check{q}_{i}^{3}}{24\left(1+\check{q}_{i}\right)\left(1+\check{q}_{i}+\check{q}_{i}^{2}\right)}, & 0<\check{q}_{i}<\frac{1}{3}, 1 \leq i \leq 2, \\
\frac{1+12 \check{q}_{i}+12 \check{q}_{i}^{2}+36 \check{q}_{i}^{3}}{216\left(1+\check{q}_{i}\right)\left(1+\check{q}_{i}+\check{q}_{i}^{2}\right)}, & \frac{1}{3} \leq \check{q}_{i}<1,1 \leq i \leq 2,\end{cases} \\
& \Phi_{2}^{\check{q}_{i}}=\int_{0}^{\frac{1}{2}} \zeta_{1}\left|\check{q}_{i} \zeta_{1}-\frac{1}{6}\right|{ }_{0} d_{\check{q}_{i}} \zeta_{1}= \begin{cases}\frac{1-2 \check{q}_{i}-2 \check{q}_{i}^{2}}{24\left(1+\check{q}_{i}\right)\left(1+\check{q}_{i}+\check{q}_{i}^{2}\right)}, & 0<\check{q}_{i}<\frac{1}{3}, 1 \leq i \leq 2, \\
\frac{18 \check{q}_{i}+18 \check{q}_{i}^{2}-7}{216\left(1+\check{q}_{i}\right)\left(1+\check{q}_{i}+\check{q}_{i}^{2}\right)}, & \frac{1}{3} \leq \check{q}_{i}<1, \quad 1 \leq i \leq 2,\end{cases} \\
& \Phi_{3}^{\check{q}_{i}}=\int_{0}^{\frac{1}{2}} \zeta_{1}\left(1-\zeta_{1}\right)\left|\check{q}_{i} \zeta_{1}-\frac{1}{6}\right|{ }_{0} d_{\check{q}_{i}} \zeta_{1}= \begin{cases}\frac{1-2 \check{q}_{i}-2 \check{q}_{i}^{3}-4 \check{q}_{i}^{4}}{48\left(1+\check{q}_{i}\right)\left(1+q_{i}^{2}\right)\left(1+\check{q}_{i}+\check{q}_{i}^{2}\right)}, & 0<\check{q}_{i}<\frac{1}{3}, 1 \leq i \leq 2, \\
\frac{108 \check{q}_{i}^{4}+54 \check{q}_{i}^{3}+12 \check{q}_{i}^{2}+54 \check{q}_{i}-17}{1296\left(1+\check{q}_{i}\right)\left(1+\check{q}_{i}^{2}\right)\left(1+\check{q}_{i}+\check{q}_{i}^{2}\right)}, & \frac{1}{3} \leq \check{q}_{i}<1, \quad 1 \leq i \leq 2,\end{cases} \\
& \Phi_{4}^{\check{q}_{i}}=\int_{\frac{1}{2}}^{1}\left(1-\zeta_{1}\right)\left|\check{q}_{i} \zeta_{1}-\frac{5}{6}\right|{ }_{0} d_{\check{q}_{i}} \zeta_{1}= \begin{cases}\frac{-5+8 q+8 \check{q}_{i}^{2}-8 \check{q}_{i}^{3}}{24\left(1+\check{q}_{i}\right)\left(1+\check{q}_{i}+\check{q}_{i}^{2}\right)}, & 0<\check{q}_{i}<\frac{5}{6}, 1 \leq i \leq 2, \\
\frac{12 \check{q}_{i}+12 \check{q}_{i}^{2}+5}{216\left(1+\check{q}_{i}\right)\left(1+\check{q}_{i}+\check{q}_{i}^{2}\right)}, & \frac{5}{6} \leq \check{q}_{i}<1,1 \leq i \leq 2,\end{cases} \\
& \Phi_{5}^{\check{q}_{i}}=\int_{\frac{1}{2}}^{1} \zeta_{1}\left|\check{q}_{i} \zeta_{1}-\frac{5}{6}\right|_{0} d_{\check{q}_{i}} \zeta_{1}= \begin{cases}\frac{5-2 \check{q}_{i}-2 \check{q}_{i}^{2}}{8\left(1+\check{q}_{i}\right)\left(1+\check{q}_{i}+\check{q}_{i}^{2}\right)}, & 0<\check{q}_{i}<\frac{5}{6}, 1 \leq i \leq 2, \\
\frac{18 \check{q}_{i}+18 \check{q}_{i}^{2}+25}{216\left(1+\check{q}_{i}\right)\left(1+\check{q}_{i}+\check{q}_{i}^{2}\right)}, & \frac{5}{6} \leq \check{q}_{i}<1,1 \leq i \leq 2,\end{cases} \\
& \Phi_{6}^{\check{q}_{i}}=\int_{\frac{1}{2}}^{1} \zeta_{1}\left(1-\zeta_{1}\right)\left|\check{q}_{i} \zeta_{1}-\frac{5}{6}\right|{ }_{0} d_{\check{q}_{i}} \zeta_{1} \\
& = \begin{cases}\frac{5-2 \check{q}_{i}+28 q_{k}^{2}-2 \check{q}_{i}^{3}-12 \check{q}_{i}^{4}}{48\left(1+\check{q}_{i}\right)\left(1+q_{i}^{2}\right)\left(1+\check{q}_{i}+\check{q}_{i}^{2}\right)}, & 0<\check{q}_{i}<\frac{5}{6}, 1 \leq i \leq 2, \\
\frac{108 \check{q}_{i}^{4}-54 \check{q}_{i}^{3}+96 \check{q}_{i}^{2}-54 \check{q}_{i}+115}{1296\left(1+\check{q}_{i}\right)\left(1+\check{q}_{i}^{2}\right)\left(1+\check{q}_{i}+\check{q}_{i}^{2}\right)}, & \frac{5}{6} \leq \check{q}_{i}<1,1 \leq i \leq 2,\end{cases} \\
& =\left(\Phi_{1}^{\check{q}_{1}}+\Phi_{4}^{\check{q}_{1}}\right)\left|\frac{\eta_{1}, \eta_{3} \partial_{\check{q}_{1}, \check{q}_{2}}^{2} \mathcal{G}\left(\eta_{1}, \eta_{3}+\zeta_{2} \mathcal{F}_{\sigma, \lambda}^{g}\left(\eta_{4}-\eta_{3}\right)\right)}{\eta_{1} \partial_{\check{q}_{1}} \zeta_{1 \eta_{3}} \partial_{\check{q}_{2}} \zeta_{2}}\right|
\end{aligned}
$$

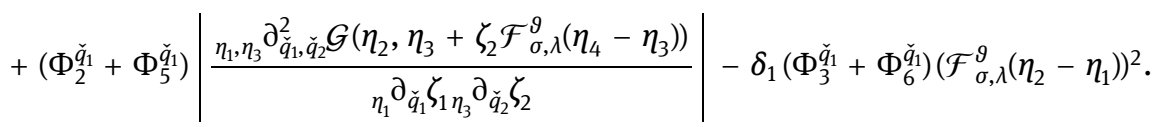


Substituting the above integrals in equation (4.1), we have

$$
\begin{aligned}
& \leq \mathcal{F}_{\sigma, \lambda}^{\vartheta}\left(\eta_{2}-\eta_{1}\right) \mathcal{F}_{\sigma, \lambda}^{\vartheta}\left(\eta_{4}-\eta_{3}\right) \int_{0}^{1}\left|\Omega_{2}\left(\zeta_{2}, \check{q}_{2}\right)\right| \\
& \left.\left.\begin{array}{c}
\left(\Phi_{1}^{\check{q}_{1}}+\Phi_{4}^{\check{q}_{1}}\right)\left|\frac{\eta_{1}, \eta_{3} \partial_{\check{q}_{1}, \check{q}_{2}}^{2} \mathcal{G}\left(\eta_{1}, \eta_{3}+\zeta_{2} \mathcal{F}_{\sigma, \lambda}^{\vartheta}\left(\eta_{4}-\eta_{3}\right)\right)}{\eta_{1} \partial_{\check{q}_{1}} \zeta_{1} \partial_{3} \partial_{\check{q}_{2}} \zeta_{2}}\right| \\
+\left(\Phi_{2}^{\check{q}_{1}}+\Phi_{5}^{\check{q}_{1}}\right)\left|\frac{\eta_{1}, \eta_{3} \partial_{\check{q}_{1}, \check{q}_{2}}^{2} \mathcal{G}\left(\eta_{2}, \eta_{3}+\zeta_{2} \mathcal{F}_{\sigma, \lambda}^{\vartheta}\left(\eta_{4}-\eta_{3}\right)\right)}{\eta_{1} \partial_{\check{q}_{1}} \zeta_{1} \eta_{3} \partial_{\check{q}_{2}} \zeta_{2}}\right| \\
-\delta_{1}\left(\Phi_{3}^{\check{q}_{1}}+\Phi_{6}^{\check{q}_{1}}\right)\left(\mathcal{F}_{\sigma, \lambda}^{\vartheta}\left(\eta_{2}, \eta_{1}\right)\right)^{2}
\end{array}\right\}\right\}_{0} d_{\check{q}_{2}} \zeta_{2} \cdot
\end{aligned}
$$

Analogously, simple computations of $\check{q}_{2}$-integral with the aid of Definitions 2.11 and 2.12 on the right-hand side of equation (4.2), yield

$$
\begin{aligned}
& \leq \mathcal{F}_{\sigma, \lambda}^{\vartheta}\left(\eta_{2}-\eta_{1}\right) \mathcal{F}_{\sigma, \lambda}^{\vartheta}\left(\eta_{4}-\eta_{3}\right)
\end{aligned}
$$

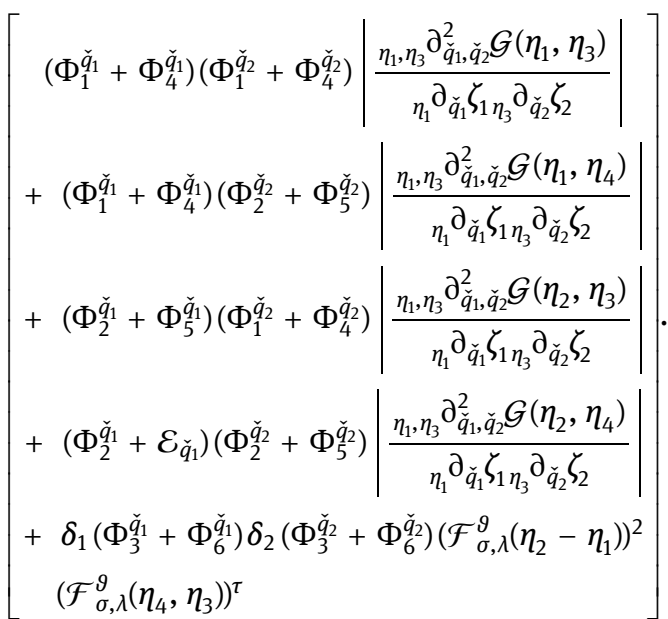

This gives the proof of Theorem 4.1.

Remark 4.1. Theorem 4.1 leads to the conclusion as follows:

I. If $\delta_{1}=\delta_{2}=0, \mathcal{F}_{\sigma, \lambda}^{\vartheta}\left(\eta_{2}-\eta_{1}\right)=\eta_{2}-\eta_{1}$ along with $\mathcal{F}_{\sigma, \lambda}^{\vartheta}$ $\left(\eta_{4}-\eta_{3}\right)=\eta_{4}-\eta_{3}$, then we get Theorem 8 of ref. [35]. II. If $\delta_{1}=\delta_{2}=0, \check{q_{1}} \mapsto 1^{-}, \check{q_{2}} \mapsto 1^{-}, \mathcal{F}_{\sigma, \lambda}^{9}\left(\eta_{2}-\eta_{1}\right)=\eta_{2}-\eta_{1}$ along with $\mathcal{F}_{\sigma, \lambda}^{9}\left(\eta_{4}-\eta_{3}\right)=\eta_{4}-\eta_{3}$, then we get Theorem 3 of ref. [41].

$$
\begin{aligned}
\left|\widetilde{\Theta}_{\mathcal{G}}\left(\eta_{1}, \eta_{2}, \eta_{3}, \eta_{4} ; \check{q}_{1}, \check{q}_{2}\right)\right| \leq & \mathcal{F}_{\sigma, \lambda}^{\vartheta}\left(\eta_{2}-\eta_{1}\right) \mathcal{F}_{\sigma, \lambda}^{\vartheta}\left(\eta_{4}-\eta_{3}\right) \int_{0}^{1} \int_{0}^{1}\left|\Omega_{1}\left(\zeta_{1}, \check{q}_{1}\right) \Omega_{2}\left(\zeta_{2}, \check{q}_{2}\right)\right| \\
& \times\left|\frac{\eta_{1}, \eta_{3} \partial_{\check{q}_{1}, \check{q}_{2}}^{2} \mathcal{G}\left(\eta_{1}+\zeta_{1}\right) \mathcal{F}_{\sigma, \lambda}^{\vartheta}\left(\eta_{2}-\eta_{1}\right), \eta_{3}+\zeta_{2} \mathcal{F}_{\sigma, \lambda}^{\vartheta}\left(\eta_{4}-\eta_{3}\right)}{{ }_{\eta_{1}} \partial_{\check{q}_{1}} \zeta_{1 \eta_{3}} \partial_{\check{q}_{2}} \zeta_{2}}\right|{ }_{0} d_{\check{q}_{1}} \zeta_{10} d_{\check{q}_{2}} \zeta_{2} .
\end{aligned}
$$

Theorem 4.2. Suppose that Assumptions (I) and (II) are satisfied. Also, if $\left|\frac{\eta_{1}, \eta_{3} \partial_{\check{q}_{1}, \breve{q}_{2}}^{2} \mathcal{G}\left(\zeta_{1}, \zeta_{2}\right)}{\eta_{1} \partial_{\check{q}_{1}} \zeta_{1} \eta_{3} \partial_{\check{q}_{2}} \zeta_{2}}\right|^{\alpha}$ is a co-ordinated generalized higher-order strongly $\Psi$-convex function for $\alpha, \beta>1$ with $\alpha^{-1}+\beta^{-1}=1$, then

$$
\begin{aligned}
& \left|\widetilde{\Theta}_{\mathcal{G}}\left(\eta_{1}, \eta_{2}, \eta_{3}, \eta_{4} ; \check{q}_{1}, \check{q}_{2}\right)\right| \\
& \leq \mathcal{F}_{\sigma, \lambda}^{\vartheta}\left(\eta_{2}-\eta_{1}\right) \mathcal{F}_{\sigma, \lambda}^{\vartheta}\left(\eta_{4}-\eta_{3}\right)\left[\left(\Phi_{7}^{\check{q}_{1}}+\Phi_{8}^{\check{q}_{1}}\right)\left(\Phi_{7}^{\check{q}_{2}}+\Phi_{8}^{\check{q}_{2}}\right)\right]^{1-\frac{1}{\alpha}} \\
& {\left[\left(\Phi_{1}^{\check{q}_{1}}+\Phi_{4}^{\check{q}_{1}}\right)\left(\Phi_{1}^{\check{q}_{2}}+\Phi_{4}^{\check{q}_{2}}\right)\left|\frac{\eta_{1}, \eta_{3} \partial_{\check{q}_{1}, \check{q}_{2}}^{2} \mathcal{G}\left(\eta_{1}, \eta_{\eta_{3}}^{\frac{1}{\alpha}}\right)}{\eta_{1} \partial_{\check{q}_{1}} \zeta_{1} \eta_{3} \partial_{\check{q}_{2}} \zeta_{2} \mid}\right|^{\alpha}\right.} \\
& +\left(\Phi_{1}^{\check{q}_{1}}+\Phi_{4}^{\check{q}_{1}}\right)\left(\Phi_{2}^{\check{q}_{2}}+\Phi_{5}^{\check{q}_{2}}\right)\left|\frac{\eta_{1}, \eta_{3} \partial_{\check{q}_{1}, \check{q}_{2}}^{2} \mathcal{G}\left(\eta_{1}, \eta_{4}\right)}{\eta_{1} \partial_{\check{q}_{1}} \zeta_{1} \eta_{3} \partial_{\check{q}_{2}} \zeta_{2}}\right|^{\alpha} \\
& \times+\left(\Phi_{2}^{\check{q}_{1}}+\Phi_{5}^{\check{q}_{1}}\right)\left(\Phi_{1}^{\check{q}_{2}}+\Phi_{4}^{\check{q}_{2}}\right)\left|\frac{\eta_{1}, \eta_{3} \partial_{\check{q}_{1}, \check{q}_{2}}^{2} \mathcal{G}\left(\eta_{2}, \eta_{3}\right)}{\eta_{1} \partial_{\check{q}_{1}} \zeta_{1} \eta_{3} \partial_{\check{q}_{2}} \zeta_{2}}\right|^{\alpha}, \\
& +\left(\Phi_{2}^{\check{q}_{1}}+\Phi_{5}^{\check{q}_{1}}\right)\left(\Phi_{2}^{\check{q}_{2}}+\Phi \check{q}_{5}^{\check{q}_{2}}\right)\left|\frac{\eta_{1}, \eta_{3} \partial_{\check{q}_{1}, \check{q}_{2}}^{2} \mathcal{G}\left(\eta_{2}, \eta_{4}\right)}{\eta_{1} \partial_{\check{q}_{1}} \zeta_{1} \eta_{3} \partial_{\check{q}_{2} \zeta_{2}}}\right|^{\alpha} \\
& +\delta_{1}\left(\Phi_{3}^{\check{q}_{1}}+\Phi_{6}^{\check{q}_{1}}\right) \delta_{2}\left(\Phi_{3}^{\check{q}_{2}}+\Phi_{6}^{\check{q}_{2}}\right)\left(\mathcal{F}_{\sigma, \lambda}^{\vartheta}\left(\eta_{2}-\eta_{1}\right)\right)^{\tau} \\
& \left(\mathcal{F}_{\sigma, \lambda}^{9}\left(\eta_{4}-\eta_{3}\right)\right)^{\tau}
\end{aligned}
$$

where

$$
\begin{gathered}
\Phi_{7}^{\check{q}_{i}}= \begin{cases}\frac{1-2 \check{q}_{i}}{12\left(1+\check{q}_{i}\right)}, & 0<\check{q}_{i}<\frac{1}{3}, 1 \leq i \leq 2, \\
\frac{6 \check{q}_{i}-1}{36\left(1+\check{q}_{i}\right)}, & \frac{1}{3} \leq \check{q}_{i}<1,1 \leq i \leq 2,\end{cases} \\
\Phi_{8}^{\check{q}_{i}}= \begin{cases}\frac{5-4 \check{q}_{i}}{12\left(1+\check{q}_{i}\right)}, & 0<\check{q}_{i}<\frac{5}{6}, 1 \leq i \leq 2, \\
\frac{4 \check{q}_{i}-5}{12\left(1+\check{q}_{i}\right)}, & \frac{5}{6} \leq \check{q}_{i}<1,1 \leq i \leq 2,\end{cases}
\end{gathered}
$$

and $\Phi_{1}^{\check{q}_{i}}, \Phi_{2}^{\check{q}_{i}}, \Phi_{3}^{\check{q}_{i}}, \Phi_{4}^{\check{q}_{i}}, \Phi_{5}^{\check{q}_{i}}$, and $\Phi_{6}^{\check{q}_{i}}$ are given in Theorem 4.1.

Proof. By means of well-known Hölder inequality, the co-ordinated generalized higher-order strongly $\Psi$-convexity of $\left|\frac{\eta_{1}, \eta_{3} \partial_{\check{q}_{1}, \check{q}_{2}}^{2} \mathcal{G}\left(\zeta_{1}, \zeta_{2}\right)}{\eta_{1} \partial_{\check{q}_{1}} \zeta_{1 \eta_{3}} \partial_{\check{q}_{2}} \zeta_{2}}\right|^{\alpha}$, and utilizing Lemma 5.1, we get 


$$
\begin{aligned}
& \leq \mathcal{F}_{\sigma, \lambda}^{g}\left(\eta_{2}-\eta_{1}\right) \mathcal{F}_{\sigma, \lambda}^{9}\left(\eta_{4}-\eta_{3}\right)\left(\int_{0}^{1} \int_{0}^{1}\left|\Omega_{1}\left(\zeta_{1}, \check{q}_{1}\right)\right|\left|\Omega_{2}\left(\zeta_{2}, \check{q}_{2}\right)\right|_{0} d_{\check{q}_{1}} \zeta_{10} d_{\check{q}_{2}} \zeta_{2}\right)^{1-\frac{1}{\alpha}}
\end{aligned}
$$

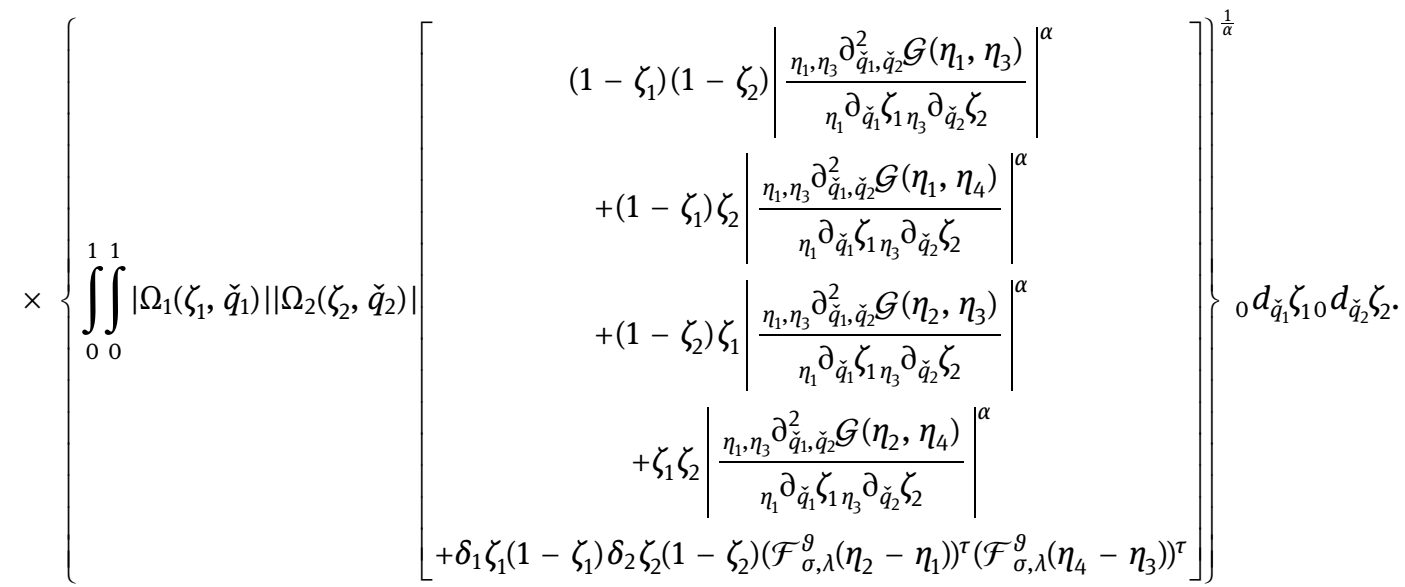

Taking into account Definitions 2.11 and 2.12, we get

$$
\begin{gathered}
\Phi_{7}^{\check{q}_{i}}=\int_{0}^{\frac{1}{2}}\left|\check{q}_{i} \zeta_{1}-\frac{1}{6}\right|_{0} d_{\check{q}_{i}} \zeta_{1}= \begin{cases}\frac{1-2 \check{q}_{i}}{12\left(1+\check{q}_{i}\right)}, & 0<\check{q}_{i}<\frac{1}{3}, 1 \leq i \leq 2, \\
\frac{6 \check{q}_{i}-1}{36\left(1+\check{q}_{i}\right)}, & \frac{1}{3} \leq \check{q}_{i}<1,1 \leq i \leq 2,\end{cases} \\
\Phi_{8}^{\check{q}_{i}}=\int_{\frac{1}{2}}^{1}\left|\check{q}_{i} \zeta_{1}-\frac{5}{6}\right|_{0} d_{\check{q}_{i}} \zeta_{1}= \begin{cases}\frac{5-4 \check{q}_{i}}{12\left(1+\check{q}_{i}\right)}, & 0<\check{q}_{i}<\frac{5}{6}, 1 \leq i \leq 2, \\
\frac{4 \check{q}_{i}-5}{12\left(1+\check{q}_{i}\right)}, & \frac{5}{6} \leq \check{q}_{i}<1,1 \leq i \leq 2,\end{cases}
\end{gathered}
$$

and derived values of $\Phi_{1}^{\check{q}_{i}}, \Phi_{2}^{\check{q}_{i}}, \Phi_{3}^{\check{q}_{i}}, \Phi_{4}^{\check{q}_{i}}, \Phi_{5}^{\check{q}_{i}}$, and $\Phi_{6}^{\check{q}_{i}}$, which have the same formulae as those given in Theorem 4.1. It follows that

$$
\begin{aligned}
& \int_{0}^{1} \int_{0}^{1}\left|\Omega_{1}\left(\zeta_{1}, \check{q}_{1}\right)\right|\left|\Omega_{2}\left(\zeta_{2}, \check{q}_{2}\right)\right|_{0} d_{\check{q}_{1}} \zeta_{10} d_{\check{q}_{2}} \zeta_{2}=\left(\int_{0}^{1}\left|\Omega_{1}\left(\zeta_{1}, \check{q}_{1}\right)\right|_{0} d_{\check{q}_{1}} \zeta_{1}\right)\left(\int_{0}^{1}\left|\Omega_{2}\left(\zeta_{2}, \check{q}_{2}\right)\right|_{0} d_{\check{q}_{2}} \zeta_{2}\right)=\left(\Phi \check{q}_{7}^{\check{q}_{1}}+\Phi_{8}^{\check{q}_{1}}\right)\left(\Phi_{7}^{\check{q}_{2}}+\Phi_{8}^{\check{q}_{2}}\right), \\
& \int_{0}^{1} \int_{0}^{1}\left(1-\zeta_{1}\right)\left(1-\zeta_{2}\right)\left|\Omega_{1}\left(\zeta_{1}, \check{q}_{1}\right)\right|\left|\Omega_{2}\left(\zeta_{2}, \check{q}_{2}\right)\right|_{0} d_{\check{q}_{1}} \zeta_{10} d_{\check{q}_{2}} \zeta_{2}=\left(\int_{0}^{1}\left(1-\zeta_{1}\right)\left|\Omega_{1}\left(\zeta_{1}, \check{q}_{1}\right)\right|_{0} d_{\breve{q}_{1}} \zeta_{1}\right)\left(\int_{0}^{1}\left(1-\zeta_{2}\right)\left|\Omega_{2}\left(\zeta_{2}, \check{q}_{2}\right)\right|_{0} d_{\check{q}_{2}} \zeta_{2}\right) \\
& =\left(\Phi_{1}^{\check{q}_{1}}+\Phi_{4}^{\check{q}_{1}}\right)\left(\Phi_{1}^{\check{q}_{2}}+\Phi_{4}^{\check{q}_{2}}\right), \\
& \int_{0}^{1} \int_{0}^{1}\left(1-\zeta_{1}\right) \zeta_{2}\left|\Omega_{1}\left(\zeta_{1}, \check{q}_{1}\right)\right|\left|\Omega_{2}\left(\zeta_{2}, \check{q}_{2}\right)\right|_{0} d_{\check{q}_{1}} \zeta_{10} d_{\check{q}_{2}} \zeta_{2}=\left(\int_{0}^{1}\left(1-\zeta_{1}\right)\left|\Omega_{1}\left(\zeta_{1}, \check{q}_{1}\right)\right|_{0} d_{\check{q}_{1}} \zeta_{1}\right)\left(\int_{0}^{1} \zeta_{2}\left|\Omega_{2}\left(\zeta_{2}, \check{q}_{2}\right)\right|_{0} d_{\check{q}_{2}} \zeta_{2}\right) \\
& =\left(\Phi_{1}^{\check{q}_{1}}+\Phi_{4}^{\check{q}_{1}}\right)\left(\Phi_{2}^{\check{q}_{2}}+\Phi_{5}^{\check{q}_{2}}\right) \text {, } \\
& \int_{0}^{1} \int_{0}^{1} \zeta_{1}\left(1-\zeta_{2}\right)\left|\Omega_{1}\left(\zeta_{1}, \check{q}_{1}\right)\right|\left|\Omega_{2}\left(\zeta_{2}, \check{q}_{2}\right)\right|_{0} d_{\check{q}_{1}} \zeta_{10} d_{\check{q}_{2}} \zeta_{2}=\left(\int_{0}^{1} \zeta_{1}\left|\Omega_{1}\left(\zeta_{1}, \check{q}_{1}\right)\right|_{0} d_{\check{q}_{1}} \zeta_{1}\right)\left(\int_{0}^{1}\left(1-\zeta_{2}\right)\left|\Omega_{2}\left(\zeta_{2}, \check{q}_{2}\right)\right|_{0} d_{\check{q}_{2}} \zeta_{2}\right) \\
& =\left(\Phi_{2}^{\check{q}_{1}}+\Phi_{5}^{\check{q}_{1}}\right)\left(\Phi_{1}^{\check{q}_{2}}+\Phi_{4}^{\check{q}_{2}}\right) \text {, }
\end{aligned}
$$




$$
\begin{aligned}
& \int_{0}^{1} \int_{0}^{1} \zeta_{1} \zeta_{2}\left|\Omega_{1}\left(\zeta_{1}, \check{q}_{1}\right)\right|\left|\Omega_{2}\left(\zeta_{2}, \check{q}_{2}\right)\right|_{0} d_{\check{q}_{1}} \zeta_{10} d_{\check{q}_{2}} \zeta_{2}=\left(\int_{0}^{1} \zeta_{1}\left|\Omega_{1}\left(\zeta_{1}, \check{q}_{1}\right)\right|_{0} d_{\check{q}_{1}} \zeta_{1}\right)\left(\int_{0}^{1} \zeta_{2}\left|\Omega_{2}\left(\zeta_{2}, \check{q}_{2}\right)\right|_{0} d_{\check{q}_{2}} \zeta_{2}\right) \\
& =\left(\Phi_{2}^{\check{q}_{2}}+\Phi_{5}^{q 2}\right)\left(\Phi_{1}^{\check{q}_{2}}+\Phi_{4}^{\check{q}_{2}}\right) \text {, } \\
& \int_{0}^{1} \int_{0}^{1} \zeta_{1} \zeta_{2}\left(1-\zeta_{1}\right)\left(1-\zeta_{2}\right)\left|\Omega_{1}\left(\zeta_{1}, \check{q}_{1}\right)\right|\left|\Omega_{2}\left(\zeta_{2}, \check{q}_{2}\right)\right|_{0} d_{\check{q}_{1}} \zeta_{10} d_{\check{q}_{2}} \zeta_{2}=\left(\int_{0}^{1} \zeta_{1}\left(1-\zeta_{1}\right)\left|\Omega_{1}\left(\zeta_{1}, \check{q}_{1}\right)\right|_{0} d_{\check{q}_{1}} \zeta_{1}\right)\left(\int_{0}^{1} \zeta_{2}\left(1-\zeta_{2}\right)\left|\Omega_{2}\left(\zeta_{2}, \check{q}_{2}\right)\right|_{0} d_{\check{q}_{2}} \zeta_{2}\right) \\
& =\left(\Phi_{3}^{\check{q}_{1}}+\Phi_{6}^{\check{q}_{1}}\right)\left(\Phi_{3}^{\check{q}_{2}}+\Phi_{6}^{\check{q}_{2}}\right) .
\end{aligned}
$$

Utilizing the values of the above $\check{q}_{1} \check{q}_{2}$-integrals, we get our required inequality.

Remark 4.2. If $\delta_{1}=\delta_{2}=0, \mathcal{F}_{\sigma, \lambda}^{9}\left(\eta_{2}-\eta_{1}\right)=\eta_{2}-\eta_{1}$ along with $\mathcal{F}_{\sigma, \lambda}^{9}\left(\eta_{4}-\eta_{3}\right)=\eta_{4}-\eta_{3}$, then Theorem 4.2 leads to Theorem 10 of ref. [35].
Theorem 4.3. Suppose that Assumptions (I) and (II) are satisfied. Also, if $\left|\frac{\eta_{1}, \eta_{3} \partial_{\ddot{q}_{1}, \breve{q}_{2}}^{2} \mathcal{G}\left(\zeta_{1}, \zeta_{2}\right)}{\eta_{1} \partial_{\check{q}_{1}} \zeta_{\eta_{3}} \partial_{\check{q}_{2}} \breve{\zeta}_{2}}\right|^{\alpha}$ is a co-ordinated generalized higher-order strongly $\Psi$-quasi-convex function for $\alpha, \beta>1$ with $\alpha^{-1}+\beta^{-1}=1$, then

$$
\begin{aligned}
& \left|\widetilde{\Theta}_{\mathcal{G}}\left(\eta_{1}, \eta_{2}, \eta_{3}, \eta_{4} ; \check{q}_{1}, \check{q}_{2}\right)\right| \leq \mathcal{F}_{\sigma, \lambda}^{9}\left(\eta_{2}-\eta_{1}\right) \mathcal{F}_{\sigma, \lambda}^{9}\left(\eta_{4}-\eta_{3}\right)\left(\left(\Phi_{7}^{\check{q}_{1}}+\Phi_{8}^{\check{q}_{1}}\right)\left(\Phi_{7}^{\check{q}_{2}}+\Phi_{8}^{\check{q}_{2}}\right)\right)^{1-\frac{1}{\alpha}}
\end{aligned}
$$

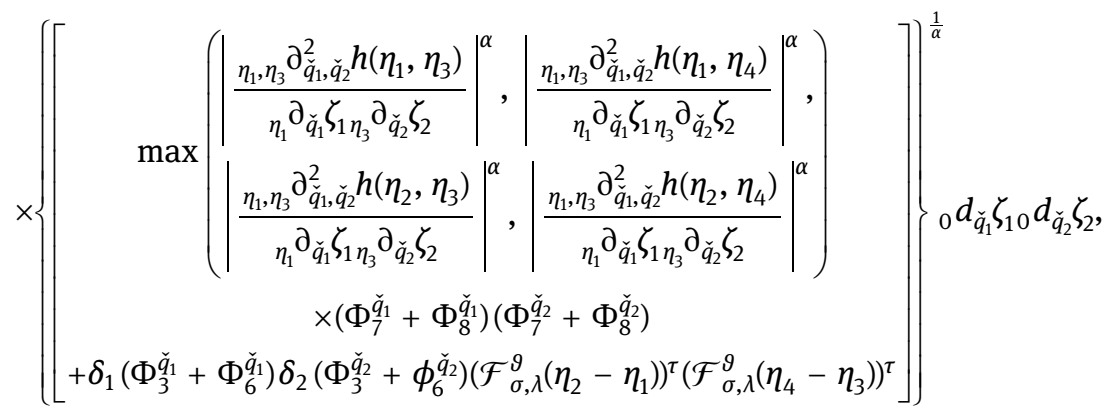

where $\Phi_{3}^{\check{q}_{i}}, \Phi_{6}^{\check{q}_{i}}$ and $\Phi_{7}^{\check{q}_{i}}, \Phi_{8}^{\check{q}_{i}}$ are given by the same expressions as described in Theorems 4.1 and 4.2.
Proof. By means of the well-known Hölder inequality, the co-ordinated generalized higher-order strongly

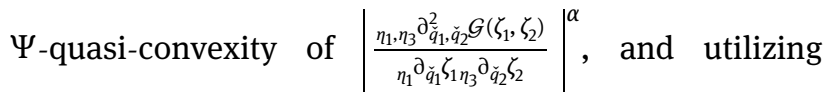
Lemma 5.1, we get

$\left|\widetilde{\Theta}_{\mathcal{G}}\left(\eta_{1}, \eta_{2}, \eta_{3}, \eta_{4} ; \check{q}_{1}, \check{q}_{2}\right)\right|$

$$
\begin{aligned}
& \leq \mathcal{F}_{\sigma, \lambda}^{9}\left(\eta_{2}-\eta_{1}\right) \mathcal{F}_{\sigma, \lambda}^{g}\left(\eta_{4}-\eta_{3}\right) \int_{0}^{1} \int_{0}^{1}\left|\Omega_{1}\left(\zeta_{1}, \check{q}_{1}\right) \Omega_{2}\left(\zeta_{2}, \check{q}_{2}\right)\right|\left|\frac{\eta_{1}, \eta_{3} \partial_{\check{q}_{1}, \check{q}_{2}}^{2} h\left(\eta_{1}+\zeta_{1}\right) \mathcal{F}_{\sigma, \lambda}^{g}\left(\eta_{2}-\eta_{1}\right), \eta_{3}+\zeta_{2} \mathcal{F}_{\sigma, \lambda}^{g}\left(\eta_{4}-\eta_{3}\right)}{\eta_{1} \partial_{\check{q}_{1}} \zeta_{1 \eta_{3}} \partial_{\check{q}_{2}} \zeta_{2}}\right| \\
& \times{ }_{0} d_{\check{q}_{1}} \zeta_{10} d_{\check{q}_{2}} \zeta_{2} . \\
& \leq \mathcal{F}_{\sigma, \lambda}^{g}\left(\eta_{2}-\eta_{1}\right) \mathcal{F}_{\sigma, \lambda}^{g}\left(\eta_{4}-\eta_{3}\right)\left(\int_{0}^{1} \int_{0}^{1}\left|\Omega_{1}\left(\zeta_{1}, \check{q}_{1}\right)\right|\left|\Omega_{2}\left(\zeta_{2}, \check{q}_{2}\right)\right|_{0} d_{\check{q}_{1}} \zeta_{10} d_{\check{q}_{2}} \zeta_{2}\right)^{1-\frac{1}{\alpha}}
\end{aligned}
$$

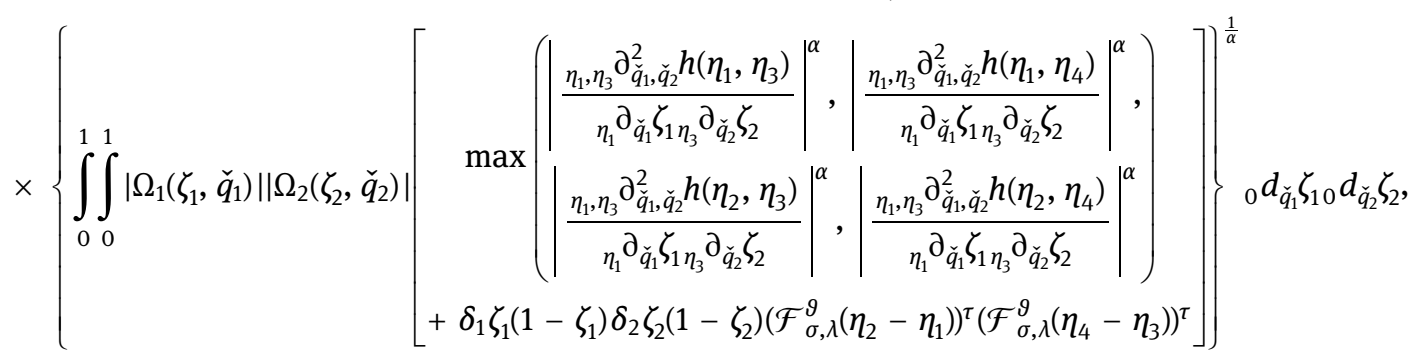


and can obtain the integral expressions of $\Phi_{3}^{\check{q}_{i}}, \Phi_{6}^{\check{q}_{i}}$ and $\Phi_{7}^{\check{q}_{i}}, \Phi_{8}^{\check{q}_{i}}$, which have the same formulae as those given in Theorems 4.1 and 4.2. This completes our result.

\section{Applications}

This study identifies some potential uses of our findings obtained in Sections 3 and 4. For appropriate and suitable choices of parameters $\sigma, \lambda$ and $\theta$ in the special functions described in (2.2), (2.3), and (2.4). Considering the Raina's function, as mentioned in (2.2), we aim to establish novel results for the hypergeometric function and Mittag-Leffler function as special cases.

\subsection{Hypergeometric function}

In this section, we assumed the following to be satisfied:
$\left(\mathrm{H}_{1}\right)$ Letting $\sigma=1$ and $\lambda=0$, and

$$
\vartheta(p)=\frac{\left(\beta_{1}\right)_{p}\left(\beta_{2}\right)_{p}}{\left(\beta_{3}\right)_{p}} \text { for } p=0,1,2, \ldots
$$

$\left(\mathrm{H}_{2}\right)$ Let a bounded sequences of positive real numbers are $\vartheta=(\vartheta(0), \ldots, \vartheta(p))$.

$\left(\mathrm{H}_{3}\right)$ Suppose that a twice partial $\check{q}_{1} \check{q}_{2}$-differentiable mapping $\mathcal{G}: \widetilde{Q} \subseteq \mathbb{R}^{2} \rightarrow \mathbb{R}$ defined on $\widetilde{Q}^{\circ}$ (the in-

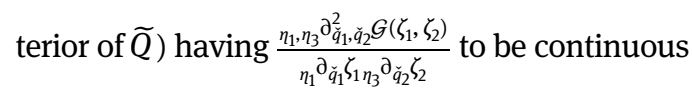
and integrable on $\left[\eta_{1}, \eta_{1}+\mathcal{F}\left(\beta_{1} ; \beta_{2} ; \beta_{3} ; \eta_{2}-\eta_{1}\right)\right] \times$ $\left[\eta_{3}, \eta_{3}+\mathcal{F}\left(\beta_{1} ; \beta_{2} ; \beta_{3} ; \eta_{4}-\eta_{3} t\right)\right] \subset \widetilde{Q}^{\circ}$ such that $\mathcal{F}\left(\beta_{1} ; \beta_{2} ; \beta_{3} ; \eta_{2}-\eta_{1}\right), \mathcal{F}\left(\beta_{1} ; \beta_{2} ; \beta_{3} ; \eta_{4}-\eta_{3}\right)>0$ for $0<\check{q}_{1}, \check{q}_{2}<1$. Then, from Lemma 5.1, Theorems 4.1, 4.2, and 4.3, the following results hold.

Lemma 5.1. Suppose that Assumptions $\left(\mathrm{H}_{1}\right),\left(\mathrm{H}_{2}\right)$ and $\left(\mathrm{H}_{3}\right)$ are satisfied, then the following equality holds:

$$
\begin{aligned}
& \widetilde{\Theta}_{\mathcal{G}}\left(\eta_{1}, \eta_{2}, \eta_{3}, \eta_{4} ; \check{q}_{1}, \check{q}_{2}\right) \\
& =\frac{1}{9}\left[\begin{array}{c}
\mathcal{G}\left(\eta_{1}, \frac{2 \eta_{3}+\mathcal{F}\left(\beta_{1} ; \beta_{2} ; \beta_{3} ; \eta_{4}-\eta_{3}\right)}{2}\right)+\mathcal{G}\left(\eta_{1}+\mathcal{F}\left(\beta_{1} ; \beta_{2} ; \beta_{3} ; \eta_{2}-\eta_{1}\right), \frac{2 \eta_{3}+\mathcal{F}\left(\beta_{1} ; \beta_{2} ; \beta_{3} ; \eta_{4}-\eta_{3}\right)}{2}\right) \\
+4 \mathcal{G}\left(\frac{2 \eta_{1}+\mathcal{F}\left(\beta_{1} ; \beta_{2} ; \beta_{3} ; \eta_{2}-\eta_{1}\right)}{2}, \frac{2 \eta_{3}+\mathcal{F}\left(\beta_{1} ; \beta_{2} ; \beta_{3} ; \eta_{4}, \eta_{3}\right)}{2}\right) \\
+\mathcal{G}\left(\frac{2 \eta_{1}+\mathcal{F}\left(\beta_{1} ; \beta_{2} ; \beta_{3} ; \eta_{2}-\eta_{1}\right)}{2}, \eta_{3}\right) \\
+\mathcal{G}\left(\frac{2 \eta_{1}+\mathcal{F}\left(\beta_{1} ; \beta_{2} ; \beta_{3} ; \eta_{2}-\eta_{1}\right)}{2}, \eta_{3}+\mathcal{F}\left(\beta_{1} ; \beta_{2} ; \beta_{3} ; \eta_{4}-\eta_{3}\right)\right)
\end{array}\right] \\
& +\frac{1}{36}\left[\begin{array}{c}
\mathcal{G}\left(\eta_{1}, \eta_{3}\right)+\mathcal{G}\left(\eta_{1}+\mathcal{F}\left(\beta_{1} ; \beta_{2} ; \beta_{3} ; \eta_{2}-\eta_{1}\right), \eta_{3}\right)+\mathcal{G}\left(\eta_{1}, \eta_{3}+\mathcal{F}\left(\beta_{1} ; \beta_{2} ; \beta_{3} ; \eta_{4}-\eta_{3}\right)\right) \\
+\mathcal{G}\left(\eta_{1}+\mathcal{F}\left(\beta_{1} ; \beta_{2} ; \beta_{3} ; \eta_{2}-\eta_{1}\right), \eta_{3}+\mathcal{F}\left(\beta_{1} ; \beta_{2} ; \beta_{3} ; \eta_{4}-\eta_{3}\right)\right)
\end{array}\right] \\
& -\frac{1}{6 \mathcal{F}\left(\beta_{1} ; \beta_{2} ; \beta_{3} ; \eta_{2}-\eta_{1}\right)} \int_{\eta_{1}}^{\eta_{1}+\mathcal{F}\left(\beta_{1} ; \beta_{2} ; \beta_{3} ; \eta_{2}-\eta_{1}\right)}\left[\begin{array}{c}
\mathcal{G}\left(x, \eta_{3}\right)+4 \mathcal{G}\left(x, \frac{2 \eta_{3}+\mathcal{F}\left(\beta_{1} ; \beta_{2} ; \beta_{3} ; \eta_{4}-\eta_{3}\right)}{2}\right) \\
+\mathcal{G}\left(x, \eta_{3}+\mathcal{F}\left(\beta_{1} ; \beta_{2} ; \beta_{3} ; \eta_{4}-\eta_{3}\right)\right)
\end{array}\right]{ }_{0} d_{\check{q}_{1} X} \\
& -\frac{1}{6 \mathcal{F}\left(\beta_{1} ; \beta_{2} ; \beta_{3} ; \eta_{4}-\eta_{3}\right)} \int_{\eta_{3}}^{\eta_{3}+\mathcal{F}\left(\beta_{1} ; \beta_{2} ; \beta_{3} ; \eta_{4}, \eta_{3}\right)}\left[\begin{array}{c}
\left.\mathcal{G}\left(\eta_{1}, y\right)+4 \mathcal{G}\left(\frac{2 \eta_{1}+\mathcal{F}\left(\beta_{1} ; \beta_{2} ; \beta_{3} ; \eta_{2}-\eta_{1}\right)}{2}, y\right)\right]_{0} d_{\check{q}_{2}} y \\
+\mathcal{G}\left(\eta_{1}+\mathcal{F}\left(\beta_{1} ; \beta_{2} ; \beta_{3} ; \eta_{2}-\eta_{1}\right), y\right)
\end{array}\right] \\
& +\frac{1}{\mathcal{F}\left(\beta_{1} ; \beta_{2} ; \beta_{3} ; \eta_{2}-\eta_{1}\right) \mathcal{F}\left(\beta_{1} ; \beta_{2} ; \beta_{3} ; \eta_{4}-\eta_{3}\right)} \int_{\eta_{1}}^{\eta_{1}+\mathcal{F}\left(\beta_{1} ; \beta_{2} ; \beta_{3} ; \eta_{2}-\eta_{1}\right)} \int_{\eta_{3}}^{\eta_{3}+\mathcal{F}\left(\beta_{1} ; \beta_{2} ; \beta_{3} ; \eta_{4}-\eta_{3}\right)} \mathcal{G}(x, y)_{0} d_{\check{q}_{2}} y_{0} d_{\check{q}_{1}} X \\
& =\mathcal{F}\left(\beta_{1} ; \beta_{2} ; \beta_{3} ; \eta_{2}-\eta_{1}\right) \mathcal{F}\left(\beta_{1} ; \beta_{2} ; \beta_{3} ; \eta_{4}-\eta_{3}\right) \int_{0}^{1} \int_{0}^{1} \Omega_{1}\left(\zeta_{1}, \check{q}_{1}\right) \Omega_{2}\left(\zeta_{2}, \check{q}_{2}\right)
\end{aligned}
$$

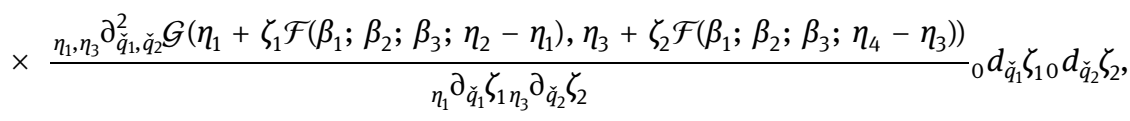


where $\Omega_{1}\left(\zeta_{1}, \check{q}_{1}\right)$ and $\Omega_{2}\left(\zeta_{1}, \check{q}_{1}\right)$ are given in (3.2) and (3.3), respectively.

Theorem 5.2. Suppose that Assumptions $\left(H_{1}\right),\left(H_{2}\right)$ and $\left(H_{3}\right)$ are satisfied. Also, if $\left|\frac{\eta_{1}, \eta_{3} \partial_{q_{1}, \breve{q}_{2}}^{2} \mathcal{G}\left(\zeta_{1}, \zeta_{2}\right)}{\eta_{1} \mathrm{\partial}_{\ddot{q}_{1}} \breve{1}_{1} \eta_{3} \mathrm{\partial}_{q_{2}} \breve{\zeta}_{2}}\right|$ is a co-ordinated generalized higher-order strongly $\Psi$-convex function, then

$$
\begin{aligned}
& \left|\widetilde{\Theta}_{\mathcal{G}}\left(\eta_{1}, \eta_{2}, \eta_{3}, \eta_{4} ; \check{q}_{1}, \check{q}_{2}\right)\right| \\
& \leq \mathcal{F}\left(\beta_{1} ; \beta_{2} ; \beta_{3} ; \eta_{2}-\eta_{1}\right) \mathcal{F}\left(\beta_{1} ; \beta_{2} ; \beta_{3} ; \eta_{4}-\eta_{3}\right)
\end{aligned}
$$

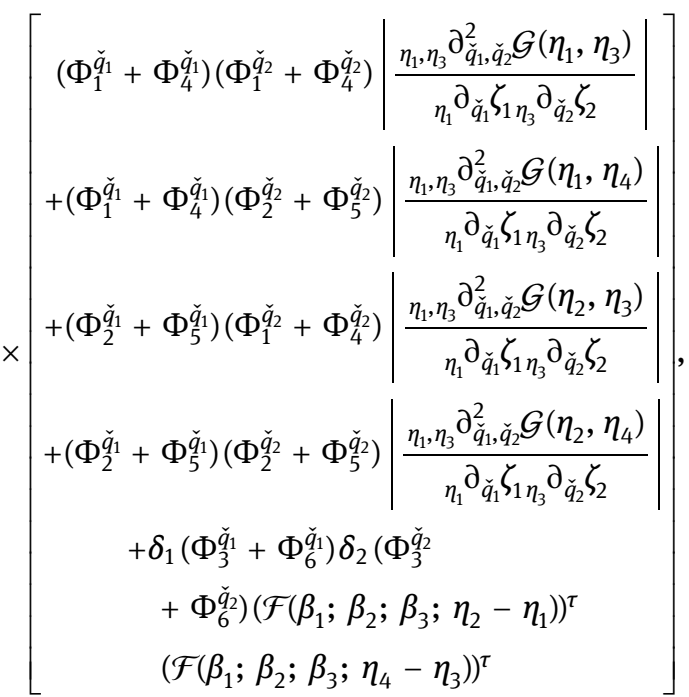

where $\Phi_{l}^{\check{q}_{i}},(l=1, \ldots, 6)$ are given in Theorem 4.1.

Theorem 5.3. Suppose that Assumptions $\left(H_{1}\right),\left(H_{2}\right)$ and $\left(H_{3}\right)$

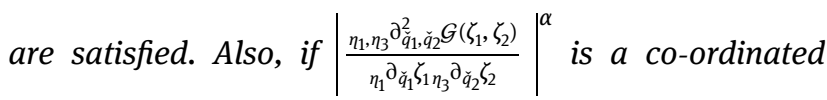

generalized higher-order strongly $\Psi$-convex function for $\alpha, \beta>1$ with $\alpha^{-1}+\beta^{-1}=1$, then

$$
\begin{aligned}
& \left|\widetilde{\Theta}_{\mathcal{G}}\left(\eta_{1}, \eta_{2}, \eta_{3}, \eta_{4} ; \check{q}_{1}, \check{q}_{2}\right)\right| \\
& \leq \mathcal{F}\left(\beta_{1} ; \beta_{2} ; \beta_{3} ; \eta_{2}-\eta_{1}\right) \mathcal{F}\left(\beta_{1} ; \beta_{2} ; \beta_{3} ;\right. \\
& \left.\eta_{4}-\eta_{3}\right)\left[\left(\Phi_{7}^{\check{q}_{1}}+\Phi_{8}^{\check{q}_{1}}\right)\left(\Phi_{7}^{\check{q}_{2}}+\Phi_{8}^{\check{q}_{2}}\right)\right]^{1-\frac{1}{\alpha}}
\end{aligned}
$$

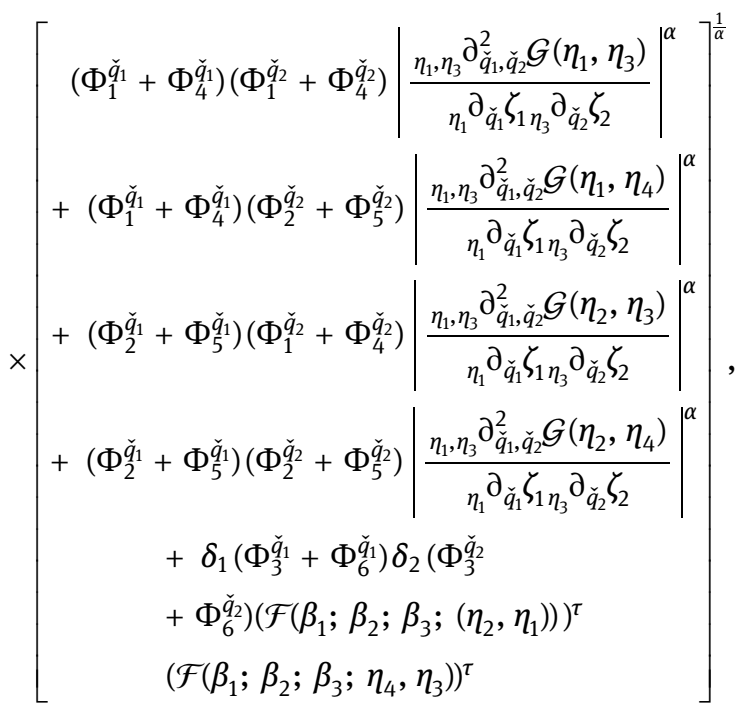

where $\Phi_{l}^{\check{q}_{i}},(l=1, \ldots, 8)$ are given in Theorems 4.1 and 4.2, respectively.

Theorem 5.4. Suppose that Assumptions $\left(H_{1}\right),\left(H_{2}\right)$ and

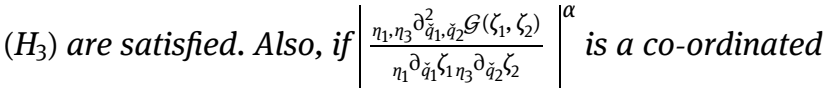
generalized higher-order strongly $\Psi$-quasi-convex function for $\alpha, \beta>1$ with $\alpha^{-1}+\beta^{-1}=1$, then

$\left|\widetilde{\Theta}_{\mathcal{G}}\left(\eta_{1}, \eta_{2}, \eta_{3}, \eta_{4} ; \check{q}_{1}, \check{q}_{2}\right)\right| \leq \mathcal{F}\left(\beta_{1} ; \beta_{2} ; \beta_{3} ; \eta_{2}-\eta_{1}\right) \mathcal{F}\left(\beta_{1} ; \beta_{2} ; \beta_{3} ; \eta_{4}-\eta_{3}\right)\left(\left(\Phi_{7}^{\check{q}_{1}}+\Phi_{8}^{\check{q}_{1}}\right)\left(\Phi_{7}^{\check{q}_{2}}+\Phi_{8}^{\check{q}_{2}}\right)\right)^{1-\frac{1}{\alpha}}$

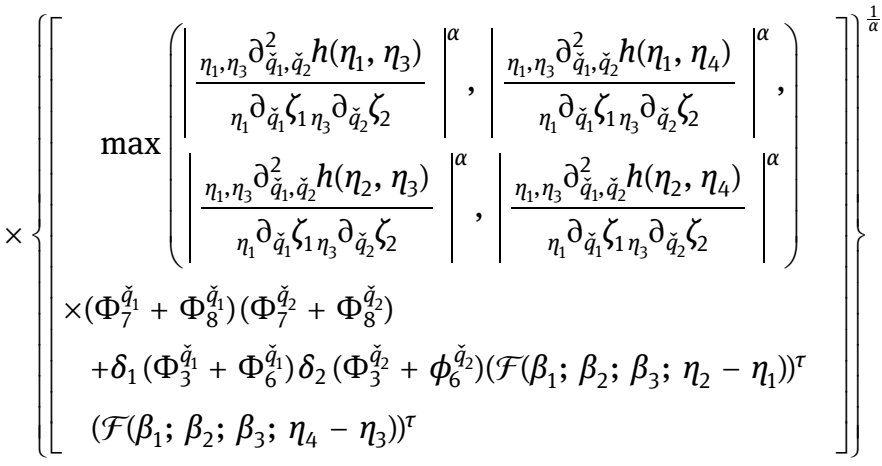

$$
\begin{aligned}
& \times{ }_{0} d_{\breve{q}_{1}} \zeta_{10} d_{\check{q}_{2}} \zeta_{2} \text {, }
\end{aligned}
$$

where $\Phi_{3}^{\breve{q}_{i}}, \Phi_{6}^{\check{q}_{i}}$ and $\Phi_{q_{i}}^{\breve{q}_{i}}, \Phi_{8}^{\check{q}_{i}}$ are given by the same expres-

sions as described in Theorems 4.1 and 4.2. 


\subsection{Mittag-Leffler function}

In this section, we assumed the following to be satisfied:

$\left(\mathrm{R}_{1}\right)$ Setting $\vartheta=(1,1, \ldots)$ having $\sigma=\beta_{1}, \mathfrak{R}\left(\beta_{1}\right)>0$ and $\lambda=1$.

$\left(\mathrm{R}_{2}\right)$ Suppose that a twice partial $\check{q}_{1} \check{q}_{2}$-differentiable mapping $\mathcal{G}: \widetilde{S} \subseteq \mathbb{R}^{2} \rightarrow \mathbb{R}$ defined on $\widetilde{S}^{\circ}$ (the in-

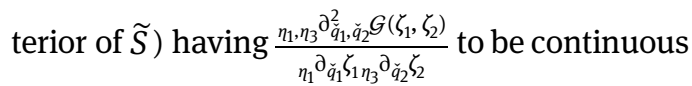

and integrable on $\left[\eta_{1}, \eta_{1}+\bar{E}_{\beta_{1}}\left(\eta_{2}-\eta_{1}\right)\right] \times$ $\left[\eta_{3}, \eta_{3}+\bar{E}_{\beta_{1}}\left(\eta_{4}-\eta_{3}\right)\right] \subset \widetilde{S}^{\circ}$ such that $\bar{E}_{\beta_{1}}\left(\eta_{2}-\eta_{1}\right)$, $\bar{E}_{\beta_{3}}\left(\eta_{4}-\eta_{3}\right)>0$ for $0<\check{q}_{1}, \check{q}_{2}<1$, then from Lemma 5.1, Theorems 4.1, 4.2 and 4.3, the following results hold.

Lemma 5.5. Suppose that Assumptions $\left(R_{1}\right)$ and $\left(R_{2}\right)$ are satisfied, then the following equality holds:

$$
\left.\begin{array}{rl}
\widetilde{\Theta}_{\mathcal{G}}\left(\eta_{1}, \eta_{2}, \eta_{3}, \eta_{4} ; \check{q}_{1}, \check{q}_{2}\right)=\frac{1}{9}\left[\begin{array}{c}
\mathcal{G}\left(\eta_{1}, \frac{2 \eta_{3}+\bar{E}_{\beta_{1}}\left(\eta_{4}-\eta_{3}\right)}{2}\right)+\mathcal{G}\left(\eta_{1}+\bar{E}_{\beta_{1}}\left(\eta_{2}-\eta_{1}\right), \frac{2 \eta_{3}+\bar{E}_{\beta_{1}}\left(\eta_{4}-\eta_{3}\right)}{2}\right) \\
+4 \mathcal{G}\left(\frac{2 \eta_{1}+\bar{E}_{\beta_{1}}\left(\eta_{2}-\eta_{1}\right)}{2}, \frac{2 \eta_{3}+\bar{E}_{\beta_{1}}\left(\eta_{4}, \eta_{3}\right)}{2}\right) \\
+\mathcal{G}\left(\frac{2 \eta_{1}+\bar{E}_{\beta_{1}}\left(\eta_{2}-\eta_{1}\right)}{2}, \eta_{3}\right)
\end{array}\right] \\
+\mathcal{G}\left(\frac{2 \eta_{1}+\bar{E}_{\beta_{1}}\left(\eta_{2}-\eta_{1}\right)}{2}, \eta_{3}+\bar{E}_{\beta_{1}}\left(\eta_{4}-\eta_{3}\right)\right)
\end{array}\right]
$$

where $\Omega_{1}\left(\zeta_{1}, \check{q}_{1}\right)$ and $\Omega_{2}\left(\zeta_{1}, \check{q}_{1}\right)$ are given in (3.2) and (3.3), respectively.
Theorem 5.1. Suppose that Assumptions $\left(R_{1}\right)$ and $\left(R_{2}\right)$ are

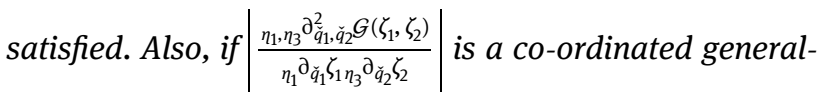
ized higher-order strongly $\Psi$-convex function, then 


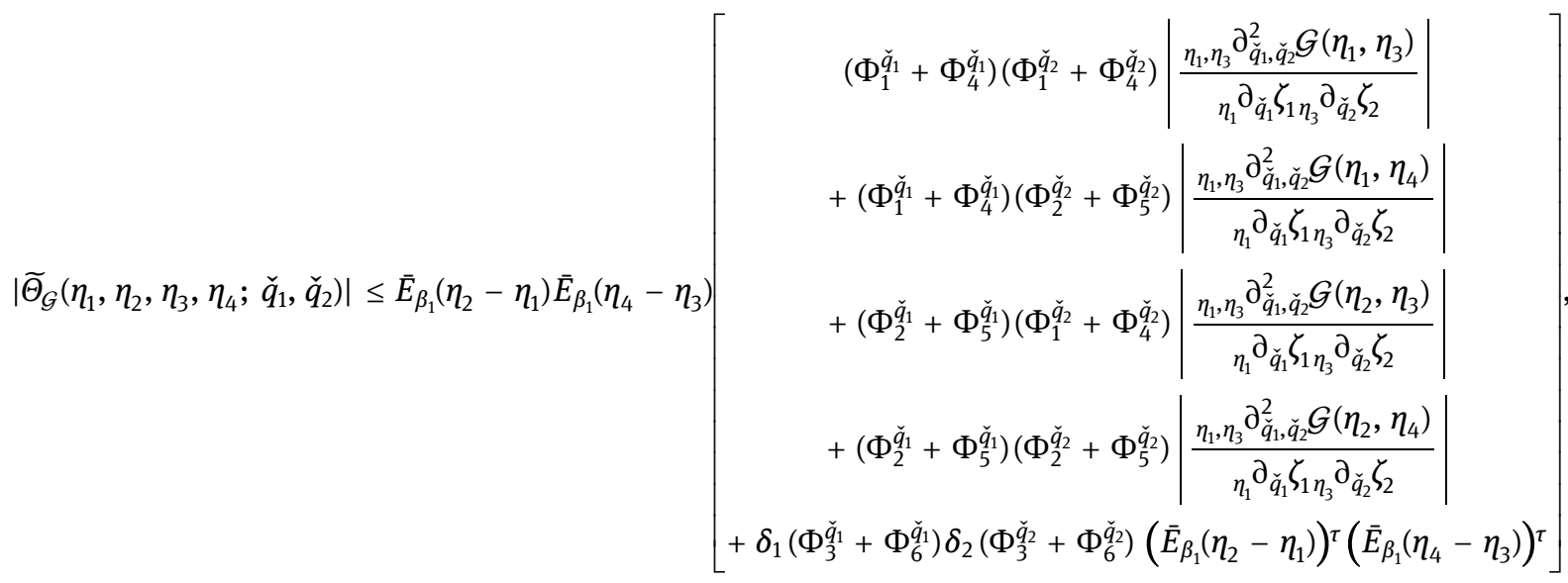

where $\Phi_{l}^{\check{q}_{i}},(l=1, \ldots, 6)$ are given in Theorem 4.1.

Theorem 5.6. Suppose that Assumptions $\left(R_{1}\right)$ and $\left(R_{2}\right)$ are generalized higher-order strongly $\Psi$-convex function for $\alpha, \beta>1$ with $\alpha^{-1}+\beta^{-1}=1$, then

satisfied. Also, if $\left|\frac{\eta_{1}, \eta_{3} \partial_{\check{q}_{1}, \breve{q}_{2}}^{2} \mathcal{G}\left(\zeta_{1}, \zeta_{2}\right)}{\eta_{1} \partial_{\check{q}_{1}} \zeta_{1 \eta_{3}} \partial_{\check{q}_{2}} \zeta_{2}}\right|^{\alpha}$ is a co-ordinated

$$
\begin{aligned}
& \left|\widetilde{\Theta}_{\mathcal{G}}\left(\eta_{1}, \eta_{2}, \eta_{3}, \eta_{4} ; \check{q}_{1}, \check{q}_{2}\right)\right| \leq \bar{E}_{\beta_{1}}\left(\eta_{2}-\eta_{1}\right) \bar{E}_{\beta_{1}}\left(\eta_{4}-\eta_{3}\right)\left[\left(\Phi_{7}^{\check{q}_{1}}+\Phi_{8}^{\check{q}_{1}}\right)\left(\Phi_{7}^{\check{q}_{2}}+\Phi_{8}^{\check{q}_{2}}\right)\right]^{1-\frac{1}{\alpha}}
\end{aligned}
$$

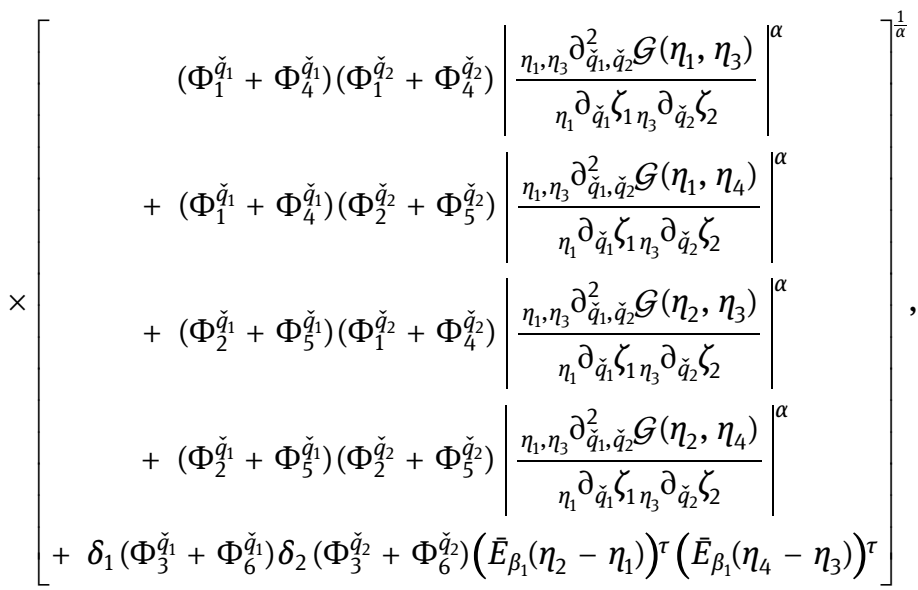

where $\Phi_{l}^{\check{q}_{i}},(l=1, \ldots, 8)$ are given in Theorems 4.1 and 4.2, respectively. generalized higher-order strongly $\Psi$-quasi-convex function for $\alpha, \beta>1$ with $\alpha^{-1}+\beta^{-1}=1$, then

Theorem 5.7. Suppose that Assumptions $\left(R_{1}\right)$ and $\left(R_{2}\right)$ are satisfied. Also, if $\left|\frac{\eta_{1}, \eta_{3} \partial_{\eta_{1}}^{2} \breve{q}_{2} \mathcal{G}\left(\zeta_{1}, \zeta_{2}\right)}{\eta_{1} \partial_{\check{q}_{1}} \zeta_{1 \eta_{3}} \partial_{\check{q}_{2}} \zeta_{2}}\right|^{\alpha}$ is a co-ordinated

$$
\begin{aligned}
& \left|\widetilde{\Theta}_{\mathcal{G}}\left(\eta_{1}, \eta_{2}, \eta_{3}, \eta_{4} ; \check{q}_{1}, \check{q}_{2}\right)\right| \leq \bar{E}_{\beta_{1}}\left(\eta_{2}-\eta_{1}\right) \bar{E}_{\beta_{1}}\left(\eta_{4}-\eta_{3}\right)\left(\left(\Phi_{7}^{\check{q}_{1}}+\Phi_{8}^{\check{q}_{1}}\right)\left(\Phi_{7}^{\check{q}_{2}}+\Phi_{8}^{\check{q}_{2}}\right)\right)^{1-\frac{1}{\alpha}}
\end{aligned}
$$

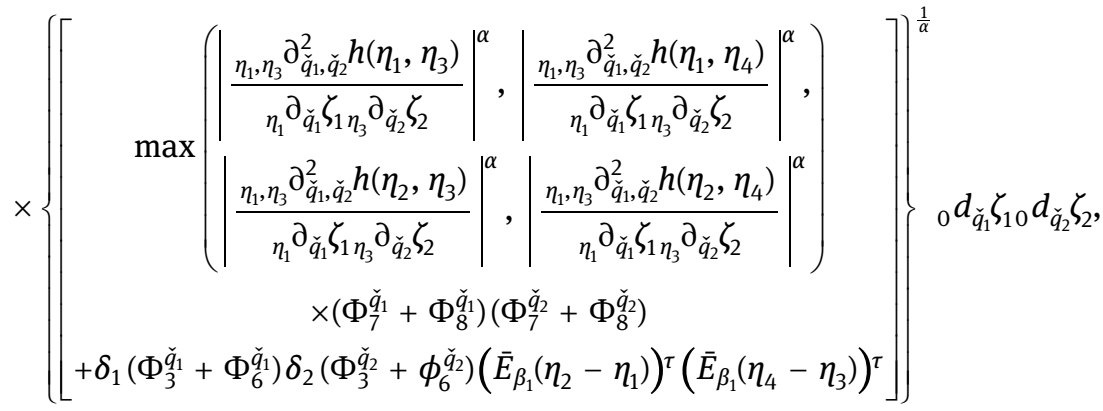


where $\Phi_{3}^{\check{q}_{i}}, \Phi_{6}^{\check{q}_{i}}$ and $\Phi_{7}^{\check{q}_{i}}, \Phi_{8}^{\check{q}_{i}}$ are given by the same expressions as described in Theorems 4.1 and 4.2.

\subsection{Conclusion}

The aim of this research is to contribute as a source of inspiration for ongoing studies. Taking into consideration $\check{q}_{1} \check{q}_{2}$-integrals, an auxiliary result has been obtained. In the special Raina's function perspective that contributes to the $\check{q}_{1} \check{q}_{2}$-identity, we constructed some new generalizations for Simpson-type inequality concerning $\check{q}_{1} \check{q}_{2}$-differentiable mappings for higher-order strongly generalized $\Psi$-convex functions. The connection of wellknown special functions (hypergeometric and MittagLeffler function) is being used to demonstrate some potential applications of our results. Furthermore, our results are extremely applicable to the solution of integral equations involving interacting $n$ bodies with mixed boundary conditions $[13,14]$.

Acknowledgments: The authors would like to express their sincere thanks to the support from the National Natural Science Foundation of China and Taif University researchers (supporting project number: TURSP-2020/155, Taif, KSA).

Funding information: This work was supported by the National Natural Science Foundation of China (Grant No. 61673169).

Author contribution: All authors contributed equally to the writing of this manuscript. All authors read and approved the final manuscript.

Conflict of interest: The authors states no conflict of interest.

\section{References}

[1] Jackson FH. On a $q$-definite integrals. Q J Pure Appl Math. 1910;41:193-203.

[2] Andrews GW. q-Series: Their development and applications in analysis: Number theory, combinatorics, physics and computer algebra. CBMS Regional Conference Series in Mathematics. Providence, RI: American Mathematical Society; 1986, p. 66.
[3] Ernst T. The history of q-calculus and a new method (Licentiate thesis). U. U. D. M; 2000.

[4] Ernst T. A method for q-calculus. J Nonlin Math Phys A Method. 2003;10:487-525.

[5] Baxter R. Exact solved models in statistical mechanics. New York, NY: Academic Press. 1982.

[6] Bettaibi N, Mezlini K. On the use Of the q-Mellin transform to solve some q-heat and q-wave equations. Int J Math Arch. 2012;3:446-55.

[7] Gauchman H. Integral inequalities in q-calculus. Comp Math Appl. 2004;47:281-300.

[8] Kac V, Cheung P. Quantum calculus, New York, NY, USA: Springer; 2002.

[9] Alp N, Sarikaya MZ, Kunt M, İşcan İ. q-Hermite Hadamard inequalities and quantum estimates for midpoint type inequalities via convex and quasi-convex functions. J King Saud Univ Sci. 2018;30:193-203.

[10] Liu WJ, Zhuang HF. Some quantum estimates of Hermite-Hadamard inequalities for convex functions. J Appl Anal Comput. 2017;7:501-22.

[11] Garg M, Chanchlani L. Kober fractional q-derivative operators. Le Matematiche. 2011;66:13-26.

[12] Purohit SD, Yadav RK. On generalized fractional q-integral operators involving the q-gauss hypergeometric function. Bull Math Anal Appl. 2010;24:35-44.

[13] Sosnovskiy LA, Sherbakov SS. A model of mechanothermodynamic entropy in tribology. Entropy. 2017;19.

[14] Shcherbakov SS. Spatial stress-strain state of tribofatigue system in roll-shaft contact zone. Strength Mater. 2013;45:35-43.

[15] Kirmaci US. Inequalities for differentiable mappings and applications to special means of real numbers to midpoint formula. Appl Math Comput. 2004;147:137-46.

[16] Dragomir SS, Agarwal RP. Two inequalities for differentiable mappings and applications to special means of real numbers and to Trapezoidal formula. Appl Math Lett. 1998;11:91-5.

[17] Kirmaci US, Özdemir ME. On some inequalities for differentiable mappings and applications to special means of real numbers and to midpoint formula. Appl Math Comput. 2004;153:361-8.

[18] Jensen JLWV. Omkonvexe Funktioner og Ulighedermellen Middelvaerdier. Nyt Tidsskr Math. 1905;16B:49-69.

[19] Jensen JLWV. Sur les fonctions convexes et les inegalités entre les valeurs moyennes. Acta Math. 1906;30:175-93.

[20] Rashid S, Noor MA, Noor KI, Safdar F, Chu YM. Hermite-Hadamard-type inequalities for the class of convex functions on time scale. Mathematics. 2019;7.

[21] Nie D, Rashid S, Akdemir AO, Baleanu D, Liu JB. On some new weighted inequalities for differentiable exponentially convex and exponentially quasi-convex functions with applications. Mathematics. 2019:7.

[22] Rashid S, Noor MA, Noor KI. Inequalities pertaining fractional approach through exponentially convex functions. Fractal Fract. 2019:3.

[23] Alomari M, Darus M, Dragomir SS. New inequalities of Simpson's type for s-convex functions with applications. RGMIA Res Rep Coll. 2009;12:1-18. 
[24] Dragomir DD, Agarwal RP, Cerone P. On Simpson's inequality and applications. J Ineq Appl. 2000;5:533-79.

[25] Sarikaya MZ, Set E, Özdemir ME. On new inequalities of Simpson-type for convex functions. Comput Math Appl. 2016;60:2191-9.

[26] Polyak BT. Existence theorems and convergence of minimizing sequences in extremum problems with restrictions. Soviet Math Dokl. 1966;7:72-5.

[27] Karamardian S. The nonlinear complementarity problems with applications. J Optim Theory Appl. 1969;4:167-81.

[28] Zu DL, Marcotte P. Co-coercivity and its role in the convergence of iterative schemes for solving variational inequalities. SIAM J Optim. 1996;6:714-26.

[29] Nikodem K, Pales ZS. Characterizations of inner product spaces by strongly convex functions. Banach 2011;1:83-7.

[30] Qu G, Li N. On the exponentially stability of primaldual gradient dynamics. IEEE Control Syst Lett. 2019;3:43-8.

[31] Rashid S, Latif MA, Hammouch Z, Chu YM. Fractional integral inequalities for strongly $\mathrm{h}$-preinvex functions for a kth order differentiable functions. Symmetry. 2019:11.

[32] Adamek M. On a problem connected with strongly convex functions. Math Inequal Appl. 2016;19:1287-93.
[33] Angulo H, Gimenez J, Moeos AM, Nikodem K. On strongly h-convex functions. Ann Funct Anal. 2011;2:85-91.

[34] Azcar A, Gimnez J, Nikodem K, Snchez JL. On strongly midconvex functions. Opuscula Math. 2011;31:15-26.

[35] Kalsoom H, Wu J, Hussain S, Latif MA. Simpson's type inequalities for co-ordinated convex functions on quantum calculus. Symmetry. 2019;11.

[36] Noor MA. Some new classes of non-convex function. Nonlinear Funct. Anal Appl. 2006;11:165-71.

[37] Raina RK. On generalized Wright's hypergeometric functions and fractional calculus operators. East Asian Math J. 2005;21:191-203.

[38] Vivas-Cortez MJ, Kashuri A, Liko R, Hernández JE. Quantum estimates of Ostrowski inequalities for generalized phi-convex Functions. Symmetry. 2019;12.

[39] Tariboon J, Ntouyas SK. Quantum integral inequalities on finite intervals. J Inequal Appl. 2014;2014.

[40] Tariboon J, Ntouyas SK. Quantum calculus on finite intervals and applications to impulsive difference equations. Adv Differ Equ. 2013;2013.

[41] Özdemir ME, Akdemir AO, Kavurmaci H, Avci M. On the Simpson's inequality for coordinated convex functions. 2011. arXiv 2010, arXiv:1101.0075. 